\title{
Scalar-field dark energy nonminimally and kinetically coupled to dark matter
}

\author{
Ryotaro Kase and Shinji Tsujikawa \\ Department of Physics, Faculty of Science, Tokyo University of Science, \\ 1-3, Kagurazaka, Shinjuku-ku, Tokyo 162-8601, Japan
}

(Dated: March 16, 2020)

\begin{abstract}
We provide a general framework for studying the dark energy cosmology in which a scalar field $\phi$ is nonminimally and kinetically coupled to Cold Dark Matter (CDM). The scalar-graviton sector is described by the action of Horndeski theories with the speed of gravitational waves equivalent to that of light, whereas CDM is treated as a perfect fluid given by a Schutz-Sorkin action. We consider two interacting Lagrangians of the forms $f_{1}(\phi, X) \rho_{c}\left(n_{c}\right)$ and $f_{2}\left(n_{c}, \phi, X\right) J_{c}^{\mu} \partial_{\mu} \phi$, where $X=-\partial^{\mu} \phi \partial_{\mu} \phi / 2, \rho_{c}$ and $n_{c}$ are the energy density and number density of CDM respectively, and $J_{c}^{\mu}$ is a vector field related to the CDM four velocity. We derive the scalar perturbation equations of motion without choosing any special gauges and identify conditions for the absence of ghosts and Laplacian instabilities on scales deep inside the sound horizon. Applying a quasi-static approximation in a gauge-invariant manner, we also obtain the effective gravitational couplings felt by $\mathrm{CDM}$ and baryons for the modes relevant to the linear growth of large-scale structures. In particular, the $n_{c}$ dependence in the coupling $f_{2}$ gives rise to an interesting possibility for realizing the gravitational coupling with CDM weaker than the Newton gravitational constant $G$.
\end{abstract}

\section{INTRODUCTION}

In spite of the numerous observational evidence for the existence of dark energy and dark matter, the origins of them have not been identified yet. Dark energy is responsible for today's cosmic acceleration [1-3], whereas dark matter is the main source for the growth of large-scale structures [4, 5]. The standard cosmological paradigm is known as the $\Lambda$-Cold Dark Matter $(\Lambda \mathrm{CDM})$ model, in which dark energy and dark matter are attributed to the cosmological constant and weakly interacting nonrelativistic particles, respectively. The $\Lambda$ CDM model is overall consistent with the current observational data, but there have been tensions for today's Hubble constant $H_{0}$ and the amplitude $\sigma_{8}$ of matter density contrast between the high- and low-redshift measurements [6-8].

The cosmological constant is not the only possibility for the origin of dark energy, but there are also other candidates like a time-varying scalar field $\phi$. If we allow scalar nonminimal and derivative couplings to gravity, Horndeski theories [9] are the most general scalar-tensor theories with second-order field equations [10-12]. Meanwhile, the gravitational wave (GW) event GW170817 [13] constrains the speed of gravity $c_{t}$ to be very close to that of light $c$, at the level $\left|c_{t} / c-1\right| \lesssim 10^{-15}$. If we strictly demand that $c_{t}=c$ without allowing any tuning among functions, the Horndeski Lagrangian is restricted to be of the form $L_{\mathrm{H}}=G_{2}(\phi, X)+G_{3}(\phi, X) \square \phi+G_{4}(\phi) R$, where $G_{2,3}$ are functions of $\phi$ and $X$, and $G_{4}$ is a function of $\phi$ alone multiplied by the Ricci scalar $R$ [14-20]. In this theoretical scheme, there are some dark energy models fitting to the observational data better than the $\Lambda$ CDM $[21,22]$. Nevertheless, it is still a challenging issue to alleviate the tensions of $H_{0}$ and $\sigma_{8}$ between the high- and low-redshift measurements.

From the viewpoint of particle physics, it is natural to expect that dark energy interacts with dark matter [23]. Such a coupling can potentially alleviate the coincidence problem as to why the energy densities of two dark components are of the same orders today. For a quintessence scalar field $\phi$ whose continuity equation is sourced by the term $\beta \rho_{c} \dot{\phi}$, where $\beta$ is coupling constant, $\rho_{c}$ is the CDM density, and $\dot{\phi}$ is the time derivative of $\phi$, there exists a scaling matter era characterized by a constant field density parameter $\Omega_{\phi}=2 \beta^{2} / 3$ followed by the dark energy dominated epoch [24-27]. In this model, the likelihood analysis with Cosmic Microwave Background (CMB) temperature anisotropies from Planck and other data give rise to the upper limit $\beta<0.062$ (95\% CL), with a mild peak of the marginalized posterior distribution around $\beta=0.036$ [28].

There have been also several phenomenological approaches to the interacting dark energy scenario in which the couplings with CDM are added to the continuity equations by hand [29-44]. One of such examples is to introduce the interacting term $\xi H \rho_{c}$ or $\xi H \rho_{\mathrm{DE}}$ to the right hand side of the CDM continuity equation, where $\xi$ is a coupling constant, $H$ is the Hubble expansion rate, and $\rho_{\mathrm{DE}}$ is the dark energy density. For the interaction $\xi H \rho_{\mathrm{DE}}$, the recent joint data analysis based on Planck, the direct measurement of $H_{0}$, galaxy clusterings, and cosmic shear measurements shows that the model with negative $\xi$ (i.e., the energy transfer from CDM to dark energy) significantly reduces the tensions of $H_{0}$ and $\sigma_{8}$ between CMB and low-redshift measurements below the $68 \%$ CL level [44]. A similar conclusion was also reached in Refs. [45, 46] for interacting models in which a subdominant fraction of dark matter decays after the recombination epoch.

At the covariant level, the phenomenological interactions mentioned above can render difficulties for defining physical quantities properly [47], e.g., the CDM velocity. This problem manifests itself for the dynamics of cosmological perturbations, leading to unphysical instabilities in the early Universe [36, 37]. In contrast, the Lagrangian formulation 
of coupled dark energy and dark matter with their fully covariant energy-momentum tensors does not give rise to any ambiguities for physical quantities at the perturbation level. In this vein, Pourtsidou et al. [48] and Boehmer et al. $[49,50]$ constructed interacting theories of the dark sector by using an action of the relativistic perfect fluid for CDM (see also Refs. [51-53]). The variational approach to the perfect fluid was originally advocated by Schutz and Sorkin [54] in 1977 and was further developed by Brown [55] in 1993. The corresponding action, which we call the Schutz-Sorkin action, contains an energy density $\rho$ and a vector field $J^{\mu}$ associated with the fluid four velocity, together with Lagrange multipliers. Since both scalar and vector degrees of freedom can be consistently implemented in this framework, the Schutz-Sorkin action has been used to describe perfect fluids in the late-time cosmology in scalar-tensor and vector-tensor theories [56-67].

Neglecting the dependence of entropy density $s$, the possible interacting Lagrangian between the scalar field $\phi$ and the CDM density $\rho_{c}$ is of the form $\mathcal{L}_{\text {int } 1}=-\sqrt{-g} f_{1}(\phi) \rho_{c}\left(n_{c}\right)$, where $g$ is the determinant of metric tensor $g_{\mu \nu}, f_{1}(\phi)$ is a function of $\phi$, and $\rho_{c}$ depends on the CDM number density $n_{c}[49]^{1}$. The Schutz-Sorkin Lagrangian contains the contribution $\mathcal{L}_{M}=-\sqrt{-g} \rho_{c}\left(n_{c}\right)$, so the total effective CDM density is given by $\hat{\rho}_{c}=\left(1+f_{1}\right) \rho_{c}$. As we will see later, the energy transfer between CDM and the scalar field is particularly transparent by considering continuity equations for $\hat{\rho}_{c}$ and $\rho_{\mathrm{DE}}$. Indeed, this interacting model accommodates the coupled quintessence scenario advocated in Refs. [23, 25].

Since the Schutz-Sorkin action contains a vector field $J_{c}^{\mu}$, we can also think of the scalar derivative coupling $J_{c}^{\mu} \partial_{\mu} \phi$. Indeed, the interacting Lagrangian of the form $\mathcal{L}_{\text {int2 }}=f_{2}\left(n_{c}, \phi\right) J_{c}^{\mu} \partial_{\mu} \phi$, where $f_{2}$ is a function of $n_{c}$ and $\phi$, was proposed in Ref. [50]. This coupling opened up a new window for the study of coupled dark energy. For instance, the effective gravitational interaction with matter density perturbations can be either weaker or stronger than the Newton gravitational constant on scales relevant to the linear growth of large-scale structures [59]. This is not the case for the coupled quintessence with the interacting Lagrangian $\mathcal{L}_{\text {int1 }}$ mentioned above, in that the scalar-matter interaction is always attractive. Hence the signature of different couplings can be probed from the observations of galaxy clusterings and weak lensing. In particular, it will be of interest to study whether models with the weak gravitational interaction for the large-scale structure growth, as indicated in current observations [28, 68-71], can be consistently constructed in this framework.

The couplings $f_{1}(\phi)$ and $f_{2}\left(n_{c}, \phi\right)$ can be generalized to include the dependence of field kinetic energy $X=$ $-\partial^{\mu} \phi \partial_{\mu} \phi / 2$, such that the two interacting Lagrangians are given by $\mathcal{L}_{\text {int1 }}=-\sqrt{-g} f_{1}(\phi, X) \rho_{c}\left(n_{c}\right)$ and $\mathcal{L}_{\text {int2 }}=$ $f_{2}\left(n_{c}, \phi, X\right) J_{c}^{\mu} \partial_{\mu} \phi$. The background cosmological dynamics with the first interaction was recently discussed for a canonical field with the potential $V(\phi)$ [72]. The interacting Lagrangians $\mathcal{L}_{\text {int1 }}$ and $\mathcal{L}_{\text {int2 }}$ may also be partially related to the theories in which CDM is conformally and disformally coupled to the metric $\bar{g}_{\mu \nu}$ different from the metric $g_{\mu \nu}$ felt by baryons [73-75]. So far, most of the past papers considered the canonical scalar or k-essence field for the dark energy sector, but we would like to extend the analysis to more general coupled Horndeski theories satisfying $c_{t}=c$. This allows the possibility for realizing the dark energy equation of state $w_{\text {DE }}$ smaller than $-1[21,22]$.

In this paper, we provide a general framework of coupled Horndeski scalar dark energy with two interacting Lagrangians $\mathcal{L}_{\text {int1 }}$ and $\mathcal{L}_{\text {int2 }}$ containing the $X$ dependence in $f_{1}$ and $f_{2}$. We derive the scalar perturbation equations of motion on the flat Friedmann-Lemaitre-Robertson-Walker (FLRW) background in a gauge-ready form and obtain the stability conditions in the small-scale limit. We also compute the effective gravitational couplings of CDM and baryons associated with the linear growth of large-scale structures. This general formulation will be useful to construct theoretically consistent models of coupled dark energy and to confront them with observations.

This paper is organized as follows. In Sec. II, we derive the background equations of motion on the flat FLRW spacetime and discuss how dark energy and CDM interact with each other. In Sec. III, we expand the total action up to second order in scalar perturbations and obtain the perturbation equations without fixing gauge conditions. In Sec. IV, we identify conditions for the absence of ghosts and Laplacian instabilities in the small-scale limit by choosing several different gauges and show that they are independent of the choice of gauges. In Sec. V, we apply the quasi-static approximation to the perturbations deep inside the sound horizon and derive the general expression of the effective gravitational couplings of CDM and baryons. Sec. VI is devoted to conclusions.

We use the natural unit where the speed of light $c$, the reduced Planck constant $\hbar$, and the Boltzmann constant $k_{B}$ are equivalent to 1 . The Newton gravitational constant $G$ is related to the reduced Planck mass $M_{\mathrm{pl}}$, as $G=$ $1 /\left(8 \pi M_{\mathrm{pl}}^{2}\right)$. The Greek and Latin indices denote components in space-time and in a three-dimensional space-adapted basis, respectively.

1 As in Refs. [49, 65, 66], this interacting Lagrangian can be written in the more general form $\mathcal{L}_{\text {int } 1}=-\sqrt{-g} \rho_{c}\left(n_{c}, \phi\right)$. 


\section{LAGRANGIAN FORMULATION OF COUPLED DARK ENERGY}

We consider the Lagrangian formulation of scalar-field dark energy coupled to CDM. The scalar-graviton sector is described by the action of Horndeski theories $\mathcal{S}_{\mathrm{H}}$ with the tensor propagation speed $c_{t}$ equivalent to 1 . Besides CDM, we also take baryons and radiations into account and assume that they do not have direct couplings to the scalar field $\phi$. The noninteracting matter sector of perfect fluids is described by the Schutz-Sorkin action $\mathcal{S}_{M}$ [54, 55]. For the coupled action $\mathcal{S}_{\text {int }}$ between $\mathrm{CDM}$ and $\phi$, we consider the two types of interactions mentioned in Introduction. Then, the total action is given by

$$
\mathcal{S}=\mathcal{S}_{\mathrm{H}}+\mathcal{S}_{M}+\mathcal{S}_{\text {int }}
$$

where

$$
\begin{aligned}
\mathcal{S}_{\mathrm{H}} & =\int \mathrm{d}^{4} x \sqrt{-g}\left[G_{4}(\phi) R+G_{2}(\phi, X)+G_{3}(\phi, X) \square \phi\right], \\
\mathcal{S}_{M} & =-\sum_{I=c, b, r} \int \mathrm{d}^{4} x\left[\sqrt{-g} \rho_{I}\left(n_{I}\right)+J_{I}^{\mu} \partial_{\mu} \ell_{I}\right], \\
\mathcal{S}_{\text {int }} & =-\int \mathrm{d}^{4} x \sqrt{-g} f_{1}(\phi, X) \rho_{c}\left(n_{c}\right)+\int \mathrm{d}^{4} x f_{2}\left(n_{c}, \phi, X\right) J_{c}^{\mu} \partial_{\mu} \phi .
\end{aligned}
$$

Here, $R$ is the Ricci scalar, $G_{4}$ is a function of $\phi$ alone, $G_{2,3}$ depend on both $\phi$ and $X=-\partial^{\mu} \phi \partial_{\mu} \phi / 2$, and $\square=g^{\mu \nu} \nabla_{\mu} \nabla_{\nu}$ is the d'Alembertian with the covariant derivative operator $\nabla_{\mu}$. The Schutz-Sorkin action (2.3) describes the perfect fluids of CDM, baryons, and radiations, labeled by $c, b, r$, respectively. The energy density $\rho_{I}$ is a function of each fluid number density $n_{I}$, which can be expressed in terms of the vector field $J_{I}^{\mu}$, as

$$
n_{I}=\sqrt{\frac{J_{I}^{\mu} J_{I}^{\nu} g_{\mu \nu}}{g}} .
$$

The fluid four-velocity $u_{I \mu}$ is defined by

$$
u_{I \mu} \equiv \frac{J_{I \mu}}{n_{I} \sqrt{-g}} .
$$

From Eq. (2.5), there is the relation $u_{I}^{\mu} u_{I \mu}=-1$. The scalar quantity $\ell_{I}$ in $\mathcal{S}_{M}$ is a Lagrange multiplier, with the notation of the partial derivative $\partial_{\mu} \ell_{I} \equiv \partial \ell_{I} / \partial x^{\mu}$. Throughout the paper, we do not include the contribution of entropy density $s_{I}$ per particle $[54,55]$ to the matter acton. The vector degrees of freedom are generally present in $\mathcal{S}_{M}[60,63]$, but we do not take them into account as they are irrelevant to the cosmological dynamics in scalar-tensor theories.

Now, we are considering interacting theories in which CDM is coupled to the scalar field through the dependence of both $\phi$ and $X$. The scalar quantities constructed from the one derivative $\partial_{\mu} \phi$ correspond to $X=-\partial^{\mu} \phi \partial_{\mu} \phi / 2$ and $Y=J_{c}^{\mu} \partial_{\mu} \phi$. The first interacting action in Eq. (2.4) possesses the $\phi$ and $X$ dependent coupling $f_{1}(\phi, X)$ with the CDM density $\rho_{c}\left(n_{c}\right)$. The second interacting action in Eq. (2.4) contains the derivative coupling $Y$ as the form $f_{2}\left(n_{c}, \phi, X\right) Y$, where $f_{2}$ is a general function of $n_{c}, \phi$, and $X$.

The theories in Ref. [49] can be recovered by choosing the couplings $f_{1}=f_{1}(\phi)$ and $f_{2}=0$ with $G_{4}=M_{\mathrm{pl}}^{2} / 2$, $G_{2}=X-V(\phi)$, and $G_{3}=0$, whereas the interactions in Ref. [50] correspond to $f_{1}=0$ and $f_{2}=f_{2}\left(n_{c}, \phi\right)$. Our analysis encompasses these two coupled dark energy theories as special cases. We note that the analysis can be extended to more general couplings ${ }^{2}$ containing the nonlinear terms in $Y$, e.g., $f_{2}\left(n_{c}, \phi, X, Y\right)[48,51-53]$. The theories in which CDM is conformally and disformally coupled to the metric $\bar{g}_{\mu \nu}=A(\phi, X) g_{\mu \nu}+B(\phi, X) \partial_{\mu} \phi \partial_{\nu} \phi$ [73-75] may be accommodated in this extended class since the term $J_{c}^{\mu} J_{c}^{\nu} \bar{g}_{\mu \nu}$ in the definition of Eq. (2.5) generates a nonlinear term in $Y$. In this paper we focus on the interacting theories containing the linear term in $Y$, leaving more general couplings for a future work.

Variation of the action $(2.1)$ with respect to $\ell_{I}$ leads to

$$
\partial_{\mu} J_{I}^{\mu}=0, \quad \text { for } \quad I=c, b, r .
$$

\footnotetext{
${ }^{2}$ We thank Edmund Copeland for pointing out this issue after the initial submission of our paper on arXiv.
} 
Varying the action (2.1) with respect to $J_{I}^{\mu}$ and using the relation $\partial n_{I} / \partial J_{I}^{\mu}=J_{I \mu} /\left(n_{I} g\right)$, it follows that

$$
\begin{gathered}
\partial_{\mu} \ell_{c}=u_{c \mu} \rho_{c, n_{c}}\left(1+f_{1}\right)-\frac{1}{\sqrt{-g}} u_{c \mu} f_{2, n_{c}} J_{c}^{\alpha} \partial_{\alpha} \phi+f_{2} \partial_{\mu} \phi, \\
\partial_{\mu} \ell_{I}=u_{I \mu} \rho_{I, n_{I}}, \quad \text { for } I=b, r,
\end{gathered}
$$

where $\rho_{I, n_{I}} \equiv \partial \rho_{I} / \partial n_{I}$ and $f_{2, n_{c}} \equiv \partial f_{2} / \partial n_{c}$.

\section{A. Background equations of motion}

We derive the field equations of motion on the flat FLRW background given by the line element

$$
\mathrm{d} s^{2}=-N^{2}(t) \mathrm{d} t^{2}+a^{2}(t) \delta_{i j} \mathrm{~d} x^{i} \mathrm{~d} x^{j}
$$

where $N(t)$ is the lapse, $a(t)$ is the scale factor, and $t$ is the cosmic time. Since the fluid four-velocity in its rest frame is given by $u_{I}^{\mu}=\left(N^{-1}, 0,0,0\right)$, Eq. (2.6) gives the relation $J_{I}^{0}=n_{I} \sqrt{-g} u_{I}^{0}$, i.e.,

$$
J_{I}^{0}=n_{I} a^{3} .
$$

From Eq. (2.7), we obtain

$$
\mathcal{N}_{I} \equiv J_{I}^{0}=n_{I} a^{3}=\text { constant }
$$

which means that the particle number $\mathcal{N}_{I}$ of each matter species is conserved.

On the background (2.10), the action (2.1) reduces to

$$
\begin{aligned}
\mathcal{S}= & \int \mathrm{d}^{4} x\left\{\frac{a^{3}}{N}\left[N^{2} G_{2}(\phi, X)-\left(\ddot{\phi}+3 \frac{\dot{a}}{a} \dot{\phi}\right) G_{3}(\phi, X)+6\left(\frac{\ddot{a}}{a}+\frac{\dot{a}^{2}}{a^{2}}\right) G_{4}(\phi)\right]+\frac{\dot{N}}{N^{2}} a^{3}\left[\dot{\phi} G_{3}(\phi, X)-6 \frac{\dot{a}}{a} G_{4}(\phi)\right]\right\} \\
& -\int \mathrm{d}^{4} x\left[N a^{3}\left\{\left[1+f_{1}(\phi, X)\right] \rho_{c}+\rho_{b}+\rho_{r}\right\}+a^{3}\left(n_{c} \dot{\ell}_{c}+n_{b} \dot{\ell}_{b}+n_{r} \dot{\ell}_{r}\right)-n_{c} a^{3} f_{2}\left(n_{c}, \phi, X\right) \dot{\phi}\right],
\end{aligned}
$$

where a dot represents the derivative with respect to $t$. The field kinetic energy $X$ is given by

$$
X=\frac{\dot{\phi}^{2}}{2 N^{2}},
$$

which contains the $N$ dependence.

From Eq. (2.12), the number densities $n_{I}$ satisfy the differential equations $\dot{n}_{I}+3 H n_{I}=0$ (with $\left.I=c, b, r\right)$, where $H \equiv \dot{a} / a$ is the Hubble expansion rate. The pressure of each matter component is given by

$$
P_{I}\left(n_{I}\right)=n_{I} \rho_{I, n_{I}}-\rho_{I}
$$

In terms of $\rho_{I}\left(n_{I}\right)$ and $P_{I}\left(n_{I}\right)$, the conservations of particle numbers translate to the continuity equations,

$$
\dot{\rho}_{I}+3 H\left(\rho_{I}+P_{I}\right)=0 \text {. }
$$

In what follows, we will consider the perfect fluid satisfying the weak energy conditions, i.e.,

$$
\rho_{I}>0, \quad \rho_{I}+P_{I}>0 .
$$

On the background (2.10), Eqs. (2.8) and (2.9) reduce, respectively, to

$$
\begin{aligned}
& \dot{\ell}_{c}=-N \rho_{c, n_{c}}\left(1+f_{1}\right)+\left(n_{c} f_{2, n_{c}}+f_{2}\right) \dot{\phi}, \\
& \dot{\ell}_{I}=-N \rho_{I, n_{I}}, \quad \text { for } I=b, r .
\end{aligned}
$$

Varying the action (2.1) with respect to $N, a, \phi$, using Eqs. (2.18)-(2.19), and setting $N=1$ at the end, we obtain the background equations,

$$
\begin{aligned}
& \mathcal{C}_{1}+\dot{\phi}^{2} f_{1, X} \rho_{c}-n_{c} \dot{\phi}^{3} f_{2, X}-\left(1+f_{1}\right) \rho_{c}-\rho_{b}-\rho_{r}=0, \\
& \mathcal{C}_{2}-n_{c}^{2} f_{2, n_{c}} \dot{\phi}+\left(1+f_{1}\right) P_{c}+P_{b}+P_{r}=0, \\
& \mathcal{C}_{3}+\left[f_{1, \phi}-\dot{\phi}^{2} f_{1, X \phi}-\ddot{\phi}\left(f_{1, X}+\dot{\phi}^{2} f_{1, X X}\right)\right] \rho_{c}+3 H \dot{\phi} f_{1, X} P_{c} \\
& -n_{c}\left(3 H n_{c} f_{2, n_{c}}-3 \dot{\phi} \ddot{\phi} f_{2, X}+3 H \dot{\phi}^{2} n_{c} f_{2, X n_{c}}-\dot{\phi}^{3} f_{2, X \phi}-\dot{\phi} 3 \ddot{\phi} f_{2, X X}\right)=0,
\end{aligned}
$$


where

$$
\begin{aligned}
& \mathcal{C}_{1}=6 G_{4} H^{2}+G_{2}-\dot{\phi}^{2} G_{2, X}-\dot{\phi}^{2}\left(G_{3, \phi}-3 H \dot{\phi} G_{3, X}\right)+6 H \dot{\phi} G_{4, \phi}, \\
& \mathcal{C}_{2}=\mathcal{C}_{1}+2 q_{t} \dot{H}-D_{6} \ddot{\phi}+D_{7} \dot{\phi}, \\
& \mathcal{C}_{3}=2 D_{1} \ddot{\phi}+3 D_{6} \dot{H}-D_{5}+3 H D_{7},
\end{aligned}
$$

and

$$
\begin{aligned}
q_{t} & =2 G_{4}, \\
D_{1} & =\frac{1}{2} G_{2, X}+G_{3, \phi}+\frac{1}{2} \dot{\phi}^{2}\left(G_{2, X X}+G_{3, X \phi}\right)-\frac{3}{2} H \dot{\phi}\left(2 G_{3, X}+\dot{\phi}^{2} G_{3, X X}\right), \\
D_{5} & =G_{2, \phi}-\dot{\phi}^{2}\left(G_{2, X \phi}+G_{3, \phi \phi}\right)+3 H \dot{\phi}\left(\dot{\phi}^{2} G_{3, X \phi}+2 G_{4, \phi \phi}\right)+6 H^{2} G_{4, \phi}, \\
D_{6} & =-\dot{\phi}^{2} G_{3, X}-2 G_{4, \phi} \\
D_{7} & =\dot{\phi}\left(G_{2, X}+2 G_{3, \phi}+2 G_{4, \phi \phi}\right)-H\left(3 \dot{\phi}^{2} G_{3, X}+2 G_{4, \phi}\right) .
\end{aligned}
$$

As we will see in Sec. II B, the quantity $q_{t}$ is associated with the no-ghost condition of tensor perturbations. The coefficients $D_{1,5,6,7}$ appear in the second-order action of scalar perturbations derived later in Sec. III.

As long as the condition

$$
q_{s} \equiv 4 D_{1} q_{t}+3 D_{6}^{2}-2 q_{t}\left(f_{1, X}+\dot{\phi}^{2} f_{1, X X}\right) \rho_{c}+2 q_{t} n_{c} \dot{\phi}\left(3 f_{2, X}+\dot{\phi}^{2} f_{2, X X}\right) \neq 0
$$

is satisfied, Eqs. (2.21) and (2.22) can be solved for $\dot{H}$ and $\ddot{\phi}$. As we will show in Sec. IV, the absence of scalar ghosts requires that $q_{s}>0$, under which the background system does not cross the singular point at which the corresponding determinant vanishes (i.e., $q_{s}=0$ ).

We define the CDM density $\hat{\rho}_{c}$ and the pressure $\hat{P}_{c}$ containing the effect of interactions with the scalar field, as

$$
\begin{aligned}
& \hat{\rho}_{c} \equiv\left(1+f_{1}\right) \rho_{c}, \\
& \hat{P}_{c} \equiv\left(1+f_{1}\right) P_{c},
\end{aligned}
$$

together with the CDM effective equation of state,

$$
\hat{w}_{c} \equiv \frac{\hat{P}_{c}}{\hat{\rho}_{c}}=\frac{P_{c}}{\rho_{c}} .
$$

The coupling $f_{1}$ modifies both the energy density and pressure of CDM. For the pressureless dust $\left(P_{c}=0\right)$, the effective pressure $\hat{P}_{c}$ vanishes.

On using the continuity Eq. (2.16), the energy density $\hat{\rho}_{c}$ obeys

$$
\dot{\hat{\rho}}_{c}+3 H\left(\hat{\rho}_{c}+\hat{P}_{c}\right)=\frac{f_{1, \phi}+\ddot{\phi} f_{1, X}}{1+f_{1}} \hat{\rho}_{c} \dot{\phi} .
$$

We can express Eqs. (2.20) and (2.21) in the following forms,

$$
\begin{aligned}
3 M_{\mathrm{pl}}^{2} H^{2} & =\rho_{\mathrm{DE}}+\hat{\rho}_{c}+\rho_{b}+\rho_{r}, \\
M_{\mathrm{pl}}^{2}\left(2 \dot{H}+3 H^{2}\right) & =-P_{\mathrm{DE}}-\hat{P}_{c}-P_{b}-P_{r},
\end{aligned}
$$

where

$$
\begin{aligned}
& \rho_{\mathrm{DE}}=-G_{2}+\dot{\phi}^{2} G_{2, X}+\dot{\phi}^{2}\left(G_{3, \phi}-3 H \dot{\phi} G_{3, X}\right)-6 H \dot{\phi} G_{4, \phi}+3\left(M_{\mathrm{pl}}^{2}-2 G_{4}\right) H^{2}-\dot{\phi}^{2} f_{1, X} \rho_{c}+n_{c} \dot{\phi}^{3} f_{2, X}, \\
& P_{\mathrm{DE}}=G_{2}+\dot{\phi}^{2}\left(G_{3, \phi}+\ddot{\phi} G_{3, X}\right)+2 G_{4, \phi}(\ddot{\phi}+2 H \dot{\phi})+2 \dot{\phi}^{2} G_{4, \phi \phi}-\left(2 \dot{H}+3 H^{2}\right)\left(M_{\mathrm{pl}}^{2}-2 G_{4}\right)-n_{c}^{2} f_{2, n_{c}} \dot{\phi} .
\end{aligned}
$$

Differentiating Eq. (2.38) with respect to $t$ and exploiting Eq. (2.22), we obtain

$$
\dot{\rho}_{\mathrm{DE}}+3 H\left(\rho_{\mathrm{DE}}+P_{\mathrm{DE}}\right)=-\frac{f_{1, \phi}+\ddot{\phi} f_{1, X}}{1+f_{1}} \hat{\rho}_{c} \dot{\phi} .
$$


The sign on the right hand side of Eq. (2.40) is opposite to that of Eq. (2.35), showing the energy exchange between the scalar field and CDM. For the couplings $f_{1}(\phi)=e^{Q \phi / M_{\mathrm{p} 1}}-1$ and $f_{2}=0$, where $Q$ is a dimensionless constant, the right hand sides of Eqs. (2.35) and (2.40) reduce, respectively, to $Q \hat{\rho}_{c} \dot{\phi} / M_{\mathrm{pl}}$ and $-Q \hat{\rho}_{c} \dot{\phi} / M_{\mathrm{pl}}$. This corresponds to the coupled dark energy scenario originally proposed in Refs. [23, 25].

The coupling $f_{2}\left(n_{c}, \phi, X\right)$ does not give rise to its contribution to the right hand sides of Eqs. (2.35) and (2.40). This reflects the fact that the interaction induced by $f_{2}\left(n_{c}, \phi, X\right)$ corresponds to the momentum transfer [48, 51, 52]. By defining $\bar{P}_{c}=\hat{P}_{c}-n_{c}^{2} f_{2, n_{c}} \dot{\phi}$ and $\bar{P}_{\mathrm{DE}}=P_{\mathrm{DE}}+n_{c}^{2} f_{2, n_{c}} \dot{\phi}$, respectively, the right hand sides of continuity equations for CDM and dark energy acquire the terms $-3 H n_{c}^{2} f_{2, n_{c}} \dot{\phi}$ and $3 H n_{c}^{2} f_{2, n_{c}} \dot{\phi}$, respectively. Here, we do not choose these definitions of effective pressures to show explicitly that the energy transfer solely comes from the coupling $f_{1}(\phi, X)$. With $\hat{\rho}_{c}$ and $\hat{P}_{c}$ defined by Eqs. (2.32) and (2.33), the CDM effective equation of state (2.34) is also equivalent to the standard value $P_{c} / \rho_{c}$.

\section{B. Tensor perturbations}

We consider the propagation of tensor perturbations $h_{i j}$ obeying the traceless and transverse conditions $h_{i}^{i}=0$ and $\partial_{i} h_{i j}=0$ on the flat FLRW background. The perturbed line element containing $h_{i j}$ is given by

$$
\mathrm{d} s^{2}=-\mathrm{d} t^{2}+a^{2}(t)\left(\delta_{i j}+h_{i j}\right) \mathrm{d} x^{i} \mathrm{~d} x^{j} .
$$

The nonvanishing components of $h_{i j}$ can be chosen as $h_{11}=h_{1}(t, z), h_{22}=-h_{1}(t, z)$, and $h_{12}=h_{21}=h_{2}(t, z)$, where the two independent modes $h_{1}$ and $h_{2}$ depend on $t$ and $z$. We expand the total action (2.1) up to second order in $h_{1}, h_{2}$ and use the background Eqs. (2.21) and (2.22). After the integration by parts, the resulting second-order tensor action yields

$$
\mathcal{S}_{t}^{(2)}=\int \mathrm{d} t \mathrm{~d}^{3} x \sum_{i=1}^{2} \frac{a^{3}}{4} q_{t}\left[\dot{h}_{i}^{2}-\frac{c_{t}^{2}}{a^{2}}\left(\partial h_{i}\right)^{2}\right],
$$

where $q_{t}$ is defined by Eq. (2.26), and

$$
c_{t}^{2}=1
$$

The tensor ghost is absent under the condition

$$
q_{t}=2 G_{4}>0 .
$$

Since the propagation speed $c_{t}$ of tensor perturbations is equivalent to that of light, the coupled dark energy theory given by the action (2.1) is consistent with the observational bound of $c_{t}$ constrained from the GW170817 event [13]. The values of $q_{t}$ and $c_{t}^{2}$ are equivalent to those in the uncoupled theories $\left(f_{1}=0\right.$ and $\left.f_{2}=0\right)$, so the interaction between the scalar field and CDM does not affect the propagation of gravitational waves.

\section{SCALAR PERTURBATION EQUATIONS IN GAUGE-READY FORM}

In this section, we first expand the action (2.1) up to second order in scalar perturbations without choosing any particular gauges. Then, we derive linear perturbation equations of motion in the form ready for fixing any gauge conditions, i.e., the gauge-ready form [64, 76, 77].

The general perturbed line element containing four scalar metric perturbations $\alpha, \chi, \zeta, E$ is given by [78-80]

$$
\mathrm{d} s^{2}=-(1+2 \alpha) \mathrm{d} t^{2}+2 \partial_{i} \chi \mathrm{d} t \mathrm{~d} x^{i}+a^{2}(t)\left[(1+2 \zeta) \delta_{i j}+2 \partial_{i} \partial_{j} E\right] \mathrm{d} x^{i} \mathrm{~d} x^{j},
$$

where the perturbed quantities depend on $t$ and $x^{i}$. The scalar field $\phi$ is also decomposed as

$$
\phi=\bar{\phi}(t)+\delta \phi,
$$

where $\bar{\phi}(t)$ is the background value and $\delta \phi$ is the perturbation. In the following, we omit the overbar from background quantities. The perturbation of field kinetic energy $X$, expanded up to second order, is given by

$$
\delta X=\dot{\phi}(\dot{\delta \phi}-\dot{\phi} \alpha)+\frac{1}{2}\left[(\dot{\delta \phi}-2 \dot{\phi} \alpha)^{2}-\frac{1}{a^{2}}(\partial \delta \phi+\dot{\phi} \partial \chi)^{2}\right]+\mathcal{O}\left(\varepsilon^{3}\right)
$$


where $\varepsilon^{n}$ represents the $n$-th order of perturbations.

For quantities in the Schutz-Sorkin action (2.3), we decompose the temporal and spatial components of $J_{I}^{\mu}$ (with $I=c, b, r)$ into the background and perturbed parts, as

$$
J_{I}^{0}=\mathcal{N}_{I}+\delta J_{I}, \quad J_{I}^{i}=\frac{\delta^{i k} \partial_{k} \delta j_{I}}{a^{2}(t)},
$$

where $\mathcal{N}_{I}$ is the conserved particle number, and $\delta J_{I}, \delta j_{I}$ correspond to scalar perturbations. For baryons and radiations, we recall that the Lagrange multipliers $\ell_{I}$ satisfy the relations (2.9). The velocity potentials $v_{I}$ are defined by

$$
u_{I i}=-\partial_{i} v_{I}
$$

Since $J_{I i}=J_{I}^{0} g_{0 i}+J_{I}^{j} g_{i j}=\mathcal{N}_{I} \partial_{i} \chi+\partial_{i} \delta j_{I}$ at linear order in perturbations, Eq. (2.6) gives

$$
\partial_{i} \delta j_{I}=-\mathcal{N}_{I}\left(\partial_{i} \chi+\partial_{i} v_{I}\right)
$$

From Eq. (2.8), the spatial derivative of $\ell_{c}$ associated with CDM perturbations yields

$$
\partial_{i} \ell_{c}=-\left\{\rho_{c, n_{c}}(t)\left[1+f_{1}(t)\right]-n_{c}(t) f_{2, n_{c}}(t) \dot{\phi}(t)\right\} \partial_{i} v_{c}+f_{2}(t) \partial_{i} \delta \phi,
$$

whose integrated solution is

$$
\ell_{c}=\mathcal{A}(t)-\left\{\rho_{c, n_{c}}(t)\left[1+f_{1}(t)\right]-n_{c}(t) f_{2, n_{c}}(t) \dot{\phi}(t)\right\} v_{c}+f_{2}(t) \delta \phi .
$$

The time-dependent function $\mathcal{A}(t)$ is determined by the condition that $\partial_{0} \ell_{c}$ computed from Eq. (2.8) coincides with $\dot{\mathcal{A}}(t)$ at the background level. Then, it follows that

$\ell_{c}=-\int^{t}\left\{\rho_{c, n_{c}}(\tilde{t})\left[1+f_{1}(\tilde{t})\right]-\left[n_{c}(\tilde{t}) f_{2, n_{c}}(\tilde{t})+f_{2}(\tilde{t})\right] \dot{\phi}(\tilde{t})\right\} \mathrm{d} \tilde{t}-\left\{\rho_{c, n_{c}}(t)\left[1+f_{1}(t)\right]-n_{c}(t) f_{2, n_{c}}(t) \dot{\phi}(t)\right\} v_{c}+f_{2}(t) \delta \phi$.

For baryons and radiations, Eq. (2.9) gives

$$
\ell_{I}=-\int^{t} \rho_{I, n_{I}}(\tilde{t}) \mathrm{d} \tilde{t}-\rho_{I, n_{I}}(t) v_{I}, \quad \text { for } I=b, r .
$$

We define the density perturbation of each matter fluid, as

$$
\delta \rho_{I} \equiv \frac{\rho_{I, n_{I}}}{a^{3}}\left[\delta J_{I}-\mathcal{N}_{I}\left(3 \zeta+\partial^{2} E\right)\right] .
$$

Then, the perturbation of $n_{I}$, which is expanded up to second order, is given by

$$
\delta n_{I}=\frac{\delta \rho_{I}}{\rho_{I, n_{I}}}-\frac{\mathcal{N}_{I}\left(\partial v_{I}\right)^{2}}{2 a^{5}}-\left(3 \zeta+\partial^{2} E\right) \frac{\delta \rho_{I}}{\rho_{I, n_{I}}}-\frac{\mathcal{N}_{I}\left(\zeta+\partial^{2} E\right)\left(3 \zeta-\partial^{2} E\right)}{2 a^{3}}+\mathcal{O}\left(\varepsilon^{3}\right),
$$

whose first term on the right hand side shows the consistency with the left hand side.

The fluid density $\rho_{I}$, which depends on $n_{I}$, is expressed in the form

$$
\begin{aligned}
\rho_{I}\left(n_{I}\right) & =\rho_{I}+\rho_{I, n_{I}} \delta n_{I}+\frac{1}{2} \rho_{I, n_{I} n_{I}} \delta n_{I}^{2}+\mathcal{O}\left(\varepsilon^{3}\right) \\
& =\rho_{I}+\left(\rho_{I}+P_{I}\right) \frac{\delta n_{I}}{n_{I}}+\frac{1}{2}\left(\rho_{I}+P_{I}\right) c_{I}^{2}\left(\frac{\delta n_{I}}{n_{I}}\right)^{2}+\mathcal{O}\left(\varepsilon^{3}\right),
\end{aligned}
$$

where $c_{I}^{2}$ is the matter sound speed squared defined by

$$
c_{I}^{2}=\frac{P_{I, n_{I}}}{\rho_{I, n_{I}}}=\frac{n_{I} \rho_{I, n_{I} n_{I}}}{\rho_{I, n_{I}}} .
$$

In the interacting action (2.4), the coupling terms $f_{1}$ and $f_{2}$ are also expanded as

$$
\begin{aligned}
f_{1}(\phi, X)= & f_{1}+f_{1, \phi} \delta \phi+f_{1, X} \delta X+\frac{1}{2} f_{1, \phi \phi} \delta \phi^{2}+\frac{1}{2} f_{1, X X} \delta X^{2}+f_{1, X \phi} \delta X \delta \phi+\mathcal{O}\left(\varepsilon^{3}\right), \\
f_{2}\left(n_{c}, \phi, X\right)= & f_{2}+f_{2, n_{c}} \delta n_{c}+f_{2, \phi} \delta \phi+f_{2, X} \delta X+\frac{1}{2} f_{2, n_{c} n_{c}} \delta n_{c}^{2}+\frac{1}{2} f_{2, \phi \phi} \delta \phi^{2}+\frac{1}{2} f_{2, X X} \delta X^{2} \\
& +f_{2, n_{c} \phi} \delta n_{c} \delta \phi+f_{2, n_{c} X} \delta n_{c} \delta X+f_{2, X \phi} \delta X \delta \phi+\mathcal{O}\left(\varepsilon^{3}\right),
\end{aligned}
$$

where $\delta X$ and $\delta n_{c}$ are given, respectively, by Eqs. (3.3) and (3.12). 


\section{A. Second-order action}

We first expand the Horndeski action (2.2) up to quadratic order in scalar perturbations without using the background Eqs. (2.20)-(2.22). After the integration by parts, the second-order Horndeski action yields

$$
\mathcal{S}_{\mathrm{H}}^{(2)}=\int \mathrm{d} t \mathrm{~d}^{3} x\left(L_{\mathrm{H}}^{\mathrm{flat}}+L_{\mathrm{H}}^{\zeta}+L_{\mathrm{H}}^{E}\right),
$$

where

$$
\begin{aligned}
L_{\mathrm{H}}^{\mathrm{flat}}=a^{3}[ & D_{1} \dot{\delta \phi}{ }^{2}+D_{2} \frac{(\partial \delta \phi)^{2}}{a^{2}}+D_{3} \delta \phi^{2}+\left(D_{4} \dot{\delta \phi}+D_{5} \delta \phi+D_{6} \frac{\partial^{2} \delta \phi}{a^{2}}\right) \alpha-\left(D_{6} \dot{\delta} \phi-D_{7} \delta \phi\right) \frac{\partial^{2} \chi}{a^{2}} \\
& \left.+\left(\dot{\phi} D_{6}-2 H q_{t}\right) \alpha \frac{\partial^{2} \chi}{a^{2}}+\left(\dot{\phi}^{2} D_{1}+3 H \dot{\phi} D_{6}-3 H^{2} q_{t}\right) \alpha^{2}+\frac{1}{2} \mathcal{C}_{1}\left\{\frac{(\partial \chi)^{2}}{a^{2}}-\alpha^{2}\right\}\right], \\
L_{\mathrm{H}}^{\zeta}=a^{3}[ & \left\{3 D_{6} \dot{\delta \phi}-3 D_{7} \delta \phi-3\left(\dot{\phi} D_{6}-2 H q_{t}\right) \alpha+2 q_{t} \frac{\partial^{2} \chi}{a^{2}}\right\} \dot{\zeta}-3 q_{t} \dot{\zeta}^{2}+q_{t} \frac{(\partial \zeta)^{2}}{a^{2}} \\
& \left.-\left(B_{1} \delta \phi+2 q_{t} \alpha\right) \frac{\partial^{2} \zeta}{a^{2}}+3\left(\mathcal{C}_{1} \alpha+\frac{1}{2} \mathcal{C}_{2} \zeta-\mathcal{C}_{3} \delta \phi\right) \zeta\right] \\
L_{\mathrm{H}}^{E}=a^{3}[ & 2 q_{t} \ddot{\zeta}+2 B_{2} \dot{\zeta}-D_{6} \ddot{\delta} \phi-B_{3} \dot{\delta} \phi+B_{4} \delta \phi+\frac{1}{a^{3}} \frac{\mathrm{d}}{\mathrm{d} t}\left\{a^{3}\left(\dot{\phi} D_{6}-2 H q_{t}\right) \alpha\right\} \\
& \left.+\mathcal{C}_{1} \alpha+\mathcal{C}_{2}\left(\zeta-\frac{1}{2} \partial^{2} E\right)-\mathcal{C}_{3} \delta \phi\right] \partial^{2} E,
\end{aligned}
$$

with the coefficients

$$
\begin{aligned}
D_{2}= & -\frac{1}{2} G_{2, X}-G_{3, \phi}+2 H \dot{\phi} G_{3, X}+\frac{1}{2} \dot{\phi}^{2} G_{3, X \phi}+\frac{1}{2}\left(2 G_{3, X}+G_{3, X X} \dot{\phi}^{2}\right) \ddot{\phi} \\
D_{3}= & \frac{1}{2} G_{2, \phi \phi}-\frac{1}{2}\left(G_{2, X \phi \phi}+G_{3, \phi \phi \phi}\right) \dot{\phi}^{2}+\frac{3}{2}\left(G_{3, X \phi \phi} \dot{\phi}^{2}-G_{2, X \phi}-2 G_{3, \phi \phi}\right) H \dot{\phi} \\
& +\frac{3}{2}\left(\dot{\phi}^{2} G_{3, X \phi}+2 G_{4, \phi \phi}\right) \dot{H}+\frac{3}{2}\left(3 \dot{\phi}^{2} G_{3, X \phi}+4 G_{4, \phi \phi}\right) H^{2} \\
& -\left[\frac{1}{2} G_{2, X \phi}+G_{3, \phi \phi}-\frac{3}{2}\left(G_{3, X X \phi} \dot{\phi}^{2}+2 G_{3, X \phi}\right) H \dot{\phi}+\frac{1}{2}\left(G_{2, X X \phi}+G_{3, X \phi \phi}\right) \dot{\phi}^{2}\right] \ddot{\phi}, \\
D_{4}= & -\left(G_{2, X}+2 G_{3, \phi}\right) \dot{\phi}-\left(G_{2, X X}+G_{3, X \phi}\right) \dot{\phi}^{3}+3\left(3 G_{3, X} \dot{\phi}^{2}+G_{3, X X} \dot{\phi}^{4}+2 G_{4, \phi}\right) H, \\
B_{1}= & \frac{2}{\dot{\phi}} \dot{q}_{t}, \quad B_{2}=\dot{q}_{t}+3 H q_{t}, \quad B_{3}=\dot{D}_{6}+3 H D_{6}-D_{7}, \quad B_{4}=\dot{D}_{7}+3 H D_{7} .
\end{aligned}
$$

We recall that the coefficients $\mathcal{C}_{1,2,3}$ and $D_{1,5,6,7}$ are given by Eqs. (2.23)-(2.25) and Eqs. (2.27)-(2.30), respectively. The coefficients $D_{2,3,4}$ can be expressed by using $q_{t}, D_{1,5,6,7}$ and their time derivatives, as

$$
\begin{aligned}
2 \dot{\phi}^{2} D_{2}= & -2 H \dot{q}_{t}-\dot{\phi}\left(\dot{D}_{6}+H D_{6}+D_{7}\right) \\
2 \dot{\phi} D_{3}= & \dot{D}_{5}+3 H D_{5}-3 H\left(\dot{D}_{7}+3 H D_{7}\right)-3 H\left[f_{1, \phi}-f_{1, X \phi} \dot{\phi}^{2}-\left(f_{1, X}+f_{1, X X} \dot{\phi}^{2}\right) \ddot{\phi}\right] \rho_{c}-9 H^{2} \dot{\phi} f_{1, X} P_{c} \\
& +3 H n_{c}\left(3 H n_{c} f_{2, n_{c}}-3 \dot{\phi} \ddot{\phi} f_{2, X}+3 H \dot{\phi}^{2} n_{c} f_{2, n_{c} X}-\dot{\phi}^{3} f_{2, X \phi}-\dot{\phi} 3 \ddot{\phi} f_{2, X X}\right) \\
D_{4}= & -2 \dot{\phi} D_{1}-3 H D_{6},
\end{aligned}
$$

where we used Eq. (2.22) for the derivation of Eq. (3.24). The second-order Horndeski action (3.17) is written in the gauge-ready form. If we choose the flat gauge in which both $\zeta$ and $E$ vanish, we have that $L_{\mathrm{H}}^{\zeta}=0$ and $L_{\mathrm{H}}^{E}=0$. In this case, what is left in $\mathcal{S}_{\mathrm{H}}^{(2)}$ is the Lagrangian $L_{\mathrm{H}}^{\mathrm{flat}}$ alone. As we will see in Sec. IV A, we can also choose other gauges depending on the problem at hand.

We also expand the sum of actions $\mathcal{S}_{M}+\mathcal{S}_{\text {int }}$ up to quadratic order in scalar perturbations. After integrating out the fields $\delta j_{I}$ by using the relation $(3.6)$, the second-order action in the matter sector is given by the sum of $\mathcal{S}_{M}^{(2)}$ and $\mathcal{S}_{\text {int }}^{(2)}$. The former is expressed in the form

$$
\mathcal{S}_{M}^{(2)}=\int \mathrm{d} t \mathrm{~d}^{3} x\left(L_{M}^{\mathrm{flat}}+L_{M}^{\zeta}+L_{M}^{E}\right),
$$


where

$$
\begin{aligned}
L_{M}^{\mathrm{flat}}= & \sum_{I=c, b, r} a^{3}\left[\left\{\left(\rho_{I}+P_{I}\right) \frac{\partial^{2} \chi}{a^{2}}-\delta \dot{\rho}_{I}-3 H\left(1+c_{I}^{2}\right) \delta \rho_{I}\right\} v_{I}-\frac{1}{2}\left(\rho_{I}+P_{I}\right) \frac{\left(\partial v_{I}\right)^{2}}{a^{2}}-\frac{c_{I}^{2}}{2\left(\rho_{I}+P_{I}\right)} \delta \rho_{I}^{2}\right. \\
& \left.\quad-\alpha \delta \rho_{I}-\frac{1}{2} \rho_{I}\left\{\frac{(\partial \chi)^{2}}{a^{2}}-\alpha^{2}\right\}\right], \\
L_{M}^{\zeta}= & -\sum_{I=c, b, r} 3 a^{3}\left[\left(\rho_{I}+P_{I}\right) v_{I} \dot{\zeta}+\rho_{I} \alpha \zeta-\frac{1}{2} P_{I} \zeta^{2}\right], \\
L_{M}^{E}= & \sum_{I=c, b, r} a^{3}\left[\left(\rho_{I}+P_{I}\right)\left(\dot{v}_{I}-3 H c_{I}^{2} v_{I}\right)-\rho_{I} \alpha+P_{I}\left(\zeta-\frac{1}{2} \partial^{2} E\right)\right] \partial^{2} E .
\end{aligned}
$$

The latter interacting second-order action is given by

$$
\mathcal{S}_{\mathrm{int}}^{(2)}=\int \mathrm{d} t \mathrm{~d}^{3} x\left(L_{\mathrm{int} 1}^{\mathrm{flat}}+L_{\mathrm{int} 1}^{\zeta}+L_{\mathrm{int} 1}^{E}+L_{\mathrm{int} 2}^{\mathrm{flat}}+L_{\mathrm{int} 2}^{\zeta}+L_{\mathrm{int} 2}^{E}\right),
$$

where

$$
\begin{aligned}
L_{\text {int } 1}^{\text {fat }}= & a^{3}\left[\left\{\left(\rho_{c}+P_{c}\right) \frac{\partial^{2} \chi}{a^{2}}-\dot{\delta} \rho_{c}-3 H\left(1+c_{c}^{2}\right) \delta \rho_{c}\right\} f_{1} v_{c}-\frac{1}{2}\left(\rho_{c}+P_{c}\right) f_{1} \frac{\left(\partial v_{c}\right)^{2}}{a^{2}}-\frac{c_{c}^{2}}{2\left(\rho_{c}+P_{c}\right)} f_{1} \delta \rho_{c}^{2}\right. \\
& -\left\{\left(f_{1}-\dot{\phi}^{2} f_{1, X}\right) \alpha+\dot{\phi} f_{1, X} \dot{\delta \phi}+f_{1, \phi} \delta \phi\right\} \delta \rho_{c}-\rho_{c} f_{1, X}\left\{\dot{\phi} \delta \phi \frac{\partial^{2} \chi}{a^{2}}-\frac{(\partial \delta \phi)^{2}}{2 a^{2}}\right\} \\
& +\frac{1}{2}\left\{\rho_{c}\left(f_{1, X \phi}+\dot{\phi}^{2} f_{1, X X \phi}\right) \ddot{\phi}-3 H P_{c} \dot{\phi} f_{1, X \phi}-\rho_{c}\left(f_{1, \phi \phi}-\dot{\phi}^{2} f_{1, X \phi \phi}\right)\right\} \delta \phi^{2}-\rho_{c}\left(f_{1, \phi}-\dot{\phi}^{2} f_{1, X \phi}\right) \alpha \delta \phi \\
& \left.-\frac{1}{2} \rho_{c}\left(f_{1, X}+\dot{\phi}^{2} f_{1, X X}\right)(\dot{\phi} \alpha-\dot{\delta} \phi)^{2}-\frac{1}{2} \rho_{c}\left(f_{1}-\dot{\phi}^{2} f_{1, X}\right)\left\{\frac{(\partial \chi)^{2}}{a^{2}}-\alpha^{2}\right\}\right], \\
L_{\text {int } 1}^{\zeta}= & -3 a^{3}\left[\left\{\left(\rho_{c}+P_{c}\right) f_{1} v_{c}-\rho_{c} \dot{\phi} f_{1, X} \delta \phi\right\} \dot{\zeta}+\rho_{c}\left(f_{1}-\dot{\phi}^{2} f_{1, X}\right) \alpha \zeta-\frac{1}{2} P_{c} f_{1} \zeta^{2}\right. \\
& \left.+\left\{\rho_{c}\left(f_{1, \phi}-\dot{\phi}^{2} f_{1, X \phi}\right)-\rho_{c} \ddot{\phi}\left(f_{1, X}+\dot{\phi}^{2} f_{1, X X}\right)+3 H P_{c} \dot{\phi} f_{1, X}\right\} \delta \phi \zeta\right], \\
L_{\text {int } 1}^{E}= & -a^{3}\left[\left\{\left(\rho_{c}+P_{c}\right) f_{1} v_{c}-\rho_{c} \dot{\phi} f_{1, X} \delta \phi\right\} \partial^{2} \dot{E}+\rho_{c}\left(f_{1}-\dot{\phi}^{2} f_{1, X}\right) \alpha \partial^{2} E-P_{c} f_{1} \zeta \partial^{2} E+\frac{1}{2} P_{c} f_{1}\left(\partial^{2} E\right)^{2}\right. \\
& \left.+\left\{\rho_{c}\left(f_{1, \phi}-\dot{\phi}^{2} f_{1, X \phi}\right)-\rho_{c} \ddot{\phi}\left(f_{1, X}+\dot{\phi}^{2} f_{1, X X}\right)+3 H P_{c} \dot{\phi} f_{1, X}\right\} \delta \phi \partial^{2} E\right],
\end{aligned}
$$

and

$$
\begin{aligned}
L_{\text {int2 }}^{\mathrm{flat}}= & -a^{3} n_{c}\left[\left\{\left(\rho_{c}+P_{c}\right) \frac{\partial^{2} \chi}{a^{2}}-\dot{\delta \rho_{c}}-3 H\left(1+c_{c}^{2}\right) \delta \rho_{c}\right\} \frac{\dot{\phi} n_{c} f_{2, n_{c}}}{\rho_{c}+P_{c}} v_{c}-\dot{\phi} n_{c} f_{2, n_{c}} \frac{\left(\partial v_{c}\right)^{2}}{2 a^{2}}-\frac{\dot{\phi} n_{c}\left(2 f_{2, n_{c}}+n_{c} f_{2, n_{c} n_{c}}\right)}{2\left(\rho_{c}+P_{c}\right)^{2}} \delta \rho_{c}^{2}\right. \\
& -\frac{1}{\rho_{c}+P_{c}}\left\{n_{c} f_{2, n_{c}} \dot{\delta \phi}-\dot{\phi}^{2}\left(f_{2, X}+n_{c} f_{2, n_{c} X}\right)(\dot{\phi} \alpha-\dot{\delta} \phi)+\left[n_{c}\left(3 H f_{2, n_{c}}+\dot{\phi} f_{2, n_{c} \phi}\right)-f_{2, X} \dot{\phi} \ddot{\phi}\right] \delta \phi\right\} \delta \rho_{c} \\
& -\dot{\phi} f_{2, X}\left\{\dot{\phi} \delta \phi \frac{\partial^{2} \chi}{a^{2}}-\frac{(\partial \delta \phi)^{2}}{2 a^{2}}\right\}+\frac{1}{2}\left\{\dot{\phi} \ddot{\phi}\left(3 f_{2, X \phi}+\dot{\phi}^{2} f_{2, X X \phi}\right)-3 H n_{c}\left(f_{2, n_{c} \phi}+\dot{\phi}^{2} f_{2, n_{c} X \phi}\right)+\dot{\phi}^{3} f_{2, X} \phi \phi\right\} \delta \phi^{2} \\
& \left.+\dot{\phi}^{3} f_{2, X \phi} \alpha \delta \phi-\frac{1}{2} \dot{\phi}\left(3 f_{2, X}+\dot{\phi}^{2} f_{2, X X}\right)(\dot{\phi} \alpha-\dot{\delta \phi})^{2}+\frac{1}{2} \dot{\phi}^{3} f_{2, X}\left\{\frac{(\partial \chi)^{2}}{a^{2}}-\alpha^{2}\right\}\right], \\
L_{\text {int2 }}^{\zeta}= & 3 a^{3} n_{c}\left[\dot{\phi}\left(n_{c} f_{2, n_{c}} v_{c}-\dot{\phi} f_{2, X} \delta \phi\right) \dot{\zeta}-\dot{\phi}^{3} f_{2, X} \alpha \zeta-\frac{1}{2} \dot{\phi} n_{c} f_{2, n_{c}} \zeta^{2}\right. \\
& \left.-\left\{\dot{\phi} \ddot{\phi}\left(3 f_{2, X}+\dot{\phi}^{2} f_{2, X X}\right)-3 H n_{c}\left(f_{2, n_{c}}+\dot{\phi}^{2} f_{2, n_{c} X}\right)+\dot{\phi}^{3} f_{2, X \phi}\right\} \delta \phi \zeta\right], \\
L_{\text {int2 }}^{E}= & a^{3} n_{c}\left[\dot{\phi}\left(n_{c} f_{2, n_{c}} v_{c}-\dot{\phi} f_{2, X} \delta \phi\right) \partial^{2} \dot{E}-\dot{\phi} n_{c} f_{2, n_{c}} \zeta \partial^{2} E-\dot{\phi}^{3} f_{2, X} \alpha \partial^{2} E+\frac{1}{2} \dot{\phi} n_{c} f_{2, n_{c}}\left(\partial^{2} E\right)^{2}\right. \\
& \left.-\left\{\dot{\phi} \ddot{\phi}\left(3 f_{2, X}+\dot{\phi}^{2} f_{2, X X}\right)-3 H n_{c}\left(f_{2, n_{c}}+\dot{\phi}^{2} f_{2, n_{c} X}\right)+\dot{\phi}^{3} f_{2, X}\right\} \delta \phi \partial^{2} E\right] .
\end{aligned}
$$


Now, we are ready for computing the total second-order action $\mathcal{S}_{s}^{(2)}=\mathcal{S}_{\mathrm{H}}^{(2)}+\mathcal{S}_{M}^{(2)}+\mathcal{S}_{\mathrm{int}}^{(2)}$. On using the background Eqs. (2.20)-(2.22), the terms containing $\mathcal{C}_{1}, \mathcal{C}_{2}$, and $\mathcal{C}_{3}$ in Eqs. (3.18)-(3.20) cancel the sum of last contributions to Eqs. (3.27), (3.31), (3.34), last two contributions to Eqs. (3.28)-(3.29), and the terms except for $\dot{\zeta}$ and $\partial^{2} \dot{E}$ in Eqs. (3.32)-(3.33) and (3.35)-(3.36). Then, the resulting full second-order action is given by

$$
\mathcal{S}_{s}^{(2)}=\int \mathrm{d} t \mathrm{~d}^{3} x\left(L_{0}+L_{\mathrm{int}}\right),
$$

where

$$
\begin{aligned}
L_{0}= & a^{3}\left\{D_{1} \dot{\delta \phi}{ }^{2}+D_{2} \frac{(\partial \delta \phi)^{2}}{a^{2}}+D_{3} \delta \phi^{2}+\left(D_{4} \dot{\delta \phi}+D_{5} \delta \phi+D_{6} \frac{\partial^{2} \delta \phi}{a^{2}}\right) \alpha-\left(D_{6} \dot{\delta \phi}-D_{7} \delta \phi\right) \frac{\partial^{2} \chi}{a^{2}}\right. \\
& +\left(\dot{\phi} D_{6}-2 H q_{t}\right) \alpha \frac{\partial^{2} \chi}{a^{2}}+\left(\dot{\phi}^{2} D_{1}+3 H \dot{\phi} D_{6}-3 H^{2} q_{t}\right) \alpha^{2} \\
& +\sum_{I=c, b, r}\left\{\left(\rho_{I}+P_{I}\right) v_{I} \frac{\partial^{2} \chi}{a^{2}}-v_{I} \dot{\delta} \rho_{I}-3 H\left(1+c_{I}^{2}\right) v_{I} \delta \rho_{I}-\frac{\rho_{I}+P_{I}}{2 a^{2}}\left(\partial v_{I}\right)^{2}-\frac{c_{I}^{2}}{2\left(\rho_{I}+P_{I}\right)} \delta \rho_{I}^{2}-\alpha \delta \rho_{I}\right\} \\
& +\left\{3 D_{6} \dot{\delta \phi}-3 D_{7} \delta \phi-3\left(\dot{\phi} D_{6}-2 H q_{t}\right) \alpha-\sum_{I=c, b, r} 3\left(\rho_{I}+P_{I}\right) v_{I}+2 q_{t} \frac{\partial^{2} \chi}{a^{2}}\right\} \dot{\zeta}-3 q_{t} \dot{\zeta}^{2} \\
& -\left(B_{1} \delta \phi+2 q_{t} \alpha\right) \frac{\partial^{2} \zeta}{a^{2}}+q_{t} \frac{(\partial \zeta)^{2}}{a^{2}}+\left[2 q_{t} \ddot{\zeta}+2 B_{2} \dot{\zeta}-D_{6} \ddot{\delta \phi}-B_{3} \dot{\delta \phi}+B_{4} \delta \phi+\frac{1}{a^{3}} \frac{\mathrm{d}}{\mathrm{d} t}\left\{a^{3}\left(\dot{\phi} D_{6}-2 H q_{t}\right) \alpha\right\}\right. \\
& \left.\left.+\sum_{I=c, b, r}\left(\rho_{I}+P_{I}\right)\left(\dot{v}_{I}-3 H c_{I}^{2} v_{I}\right)\right] \partial^{2} E\right\}
\end{aligned}
$$

and

$$
\begin{aligned}
L_{\text {int }}= & a^{3}\left[\left(f_{1}-\frac{\dot{\phi} n_{c}^{2} f_{2, n_{c}}}{\rho_{c}+P_{c}}\right)\left\{\left(\rho_{c}+P_{c}\right) \frac{\partial^{2} \chi}{a^{2}}-\dot{\delta \rho_{c}}-3 H\left(1+c_{c}^{2}\right) \delta \rho_{c}\right\} v_{c}-\left\{\left(\rho_{c}+P_{c}\right) f_{1}-\dot{\phi} n_{c}^{2} f_{\left.2, n_{c}\right\}} \frac{\left(\partial v_{c}\right)^{2}}{2 a^{2}}\right.\right. \\
& -\left\{\left(f_{1}-\dot{\phi}^{2} f_{1, X}+\frac{\dot{\phi}^{3} n_{c}\left\{f_{2, X}+n_{c} f_{2, n_{c} X}\right\}}{\rho_{c}+P_{c}}\right) \alpha+\left(\dot{\phi} f_{1, X}-\frac{n_{c}\left\{n_{c} f_{2, n_{c}}+\dot{\phi}^{2}\left(f_{2, X}+n_{c} f_{2, n_{c} X}\right)\right\}}{\rho_{c}+P_{c}}\right) \dot{\delta \phi}\right. \\
& \left.+\left(f_{1, \phi}-\frac{n_{c}\left\{n_{c}\left(3 H f_{2, n_{c}}+\dot{\phi} f_{2, n_{c} \phi}\right)-\dot{\phi} \ddot{\phi} f_{2, X}\right\}}{\rho_{c}+P_{c}}\right) \delta \phi\right\} \delta \rho_{c}-\left(f_{1, X} \rho_{c}-\dot{\phi} n_{c} f_{2, X}\right)\left\{\dot{\phi} \delta \phi \frac{\partial^{2} \chi}{a^{2}}-\frac{(\partial \delta \phi)^{2}}{2 a^{2}}\right\} \\
& +\frac{1}{2}\left\{\rho_{c}\left(f_{1, X \phi}+\dot{\phi}^{2} f_{1, X X \phi}\right) \ddot{\phi}-n_{c}\left(3 f_{2, X \phi}+\dot{\phi}^{2} f_{2, X X \phi}\right) \dot{\phi} \ddot{\phi}-\rho_{c}\left(f_{1, \phi \phi}-\dot{\phi}^{2} f_{1, X \phi \phi}\right)-3 H P_{c} \dot{\phi} f_{1, X \phi}\right. \\
& \left.+n_{c}\left(3 H n_{c}\left\{f_{2, n_{c} \phi}+\dot{\phi}^{2} f_{2, n_{c} X \phi}\right\}-\dot{\phi}^{3} f_{2, X \phi \phi}\right)\right\} \delta \phi^{2}-\frac{1}{2\left(\rho_{c}+P_{c}\right)}\left\{f_{1} c_{c}^{2}-\frac{\dot{\phi} n_{c}^{2}\left(2 f_{2, n_{c}}+n_{c} f_{2, n_{c} n_{c}}\right)}{\rho_{c}+P_{c}}\right\} \delta \rho_{c}^{2} \\
& -\left\{\rho_{c}\left(f_{1, \phi}-\dot{\phi}^{2} f_{1, X \phi}\right)+\dot{\phi}^{3} n_{c} f_{2, X \phi}\right\} \alpha \delta \phi-\frac{1}{2}\left\{\rho_{c}\left(f_{1, X}+\dot{\phi}^{2} f_{1, X X}\right)-\dot{\phi} n_{c}\left(3 f_{2, X}+\dot{\phi}^{2} f_{2, X X}\right)\right\}(\dot{\phi} \alpha-\dot{\delta \phi})^{2} \\
& \left.-\left\{\left[\left(\rho_{c}+P_{c}\right) f_{1}-\dot{\phi} n_{c}^{2} f_{2, n_{c}}\right] v_{c}-\dot{\phi}\left(\rho_{c} f_{1, X}-\dot{\phi} n_{c} f_{2, X}\right) \delta \phi\right\}\left(3 \dot{\zeta}+\partial^{2} \dot{E}\right)\right] .
\end{aligned}
$$

The Lagrangian $L_{\text {int }}$ characterizes the interaction between CDM and the scalar field arising from nonvanishing couplings $f_{1}$ and $f_{2}$.

\section{B. Perturbation equations}

We derive all the linear perturbation equations of motion in Fourier space with the comoving wavenumber $k$. Varying the action (3.37) with respect to nondynamical fields $\alpha, \chi, v_{I}$, and $E$, it follows that

$$
\begin{aligned}
& {\left[D_{4}+\dot{\phi}\left(f_{1, X}+\dot{\phi}^{2} f_{1, X X}\right) \rho_{c}-n_{c} \dot{\phi}^{2}\left(3 f_{2, X}+\dot{\phi}^{2} f_{2, X X}\right)\right] \dot{\delta} \phi-3\left(\dot{\phi} D_{6}-2 H q_{t}\right) \dot{\zeta}} \\
& +\left[D_{5}-\left(f_{1, \phi}-\dot{\phi}^{2} f_{1, X \phi}\right) \rho_{c}-\dot{\phi}^{3} n_{c} f_{2, X \phi}\right] \delta \phi+\left[2 \dot{\phi}^{2} D_{1}+6 H \dot{\phi} D_{6}-6 H^{2} q_{t}-\dot{\phi}^{2}\left(f_{1, X}+\dot{\phi}^{2} f_{1, X X}\right) \rho_{c}\right.
\end{aligned}
$$




$$
\begin{aligned}
& \left.+\dot{\phi}^{3} n_{c}\left(3 f_{2, X}+\dot{\phi}^{2} f_{2, X X}\right)\right] \alpha+\frac{k^{2}}{a^{2}}\left[2 q_{t} \zeta-\left(\dot{\phi} D_{6}-2 H q_{t}\right)\left(\chi-a^{2} \dot{E}\right)-D_{6} \delta \phi\right]-\sum_{I=c, b, r} \delta \rho_{I} \\
& -\left[f_{1}-\dot{\phi}^{2} f_{1, X}+\frac{\dot{\phi}^{3} n_{c}\left(f_{2, X}+n_{c} f_{2, n_{c} X}\right)}{\rho_{c}+P_{c}}\right] \delta \rho_{c}=0, \\
& D_{6} \dot{\delta \phi}-2 q_{t} \dot{\zeta}-\left(D_{7}-\dot{\phi} f_{1, X} \rho_{c}+\dot{\phi}^{2} n_{c} f_{2, X}\right) \delta \phi-\left(\dot{\phi} D_{6}-2 H q_{t}\right) \alpha-\sum_{I=c, b, r}\left(\rho_{I}+P_{I}\right) v_{I} \\
& -\left[f_{1}\left(\rho_{c}+P_{c}\right)-\dot{\phi} n_{c}^{2} f_{2, n_{c}}\right] v_{c}=0, \\
& \dot{\delta} \rho_{I}+3 H\left(1+c_{I}^{2}\right) \delta \rho_{I}+3\left(\rho_{I}+P_{I}\right) \dot{\zeta}+\frac{k^{2}}{a^{2}}\left(\rho_{I}+P_{I}\right)\left(v_{I}+\chi-a^{2} \dot{E}\right)=0, \quad \text { for } \quad I=c, b, r, \\
& 2 q_{t} \ddot{\zeta}+2 B_{2} \dot{\zeta}-D_{6} \ddot{\delta \phi}-\left(B_{3}+\dot{\phi} f_{1, X} \rho_{c}-\dot{\phi}^{2} n_{c} f_{2, X}\right) \dot{\delta \phi}+\left[B_{4}-\left\{\dot{\phi}^{2} f_{1, X \phi}+\ddot{\phi}\left(f_{1, X}+\dot{\phi}^{2} f_{1, X X}\right)\right\} \rho_{c}\right. \\
& \left.+3 H P_{c} \dot{\phi} f_{1, X}+\dot{\phi} n_{c}\left\{\ddot{\phi}\left(2 f_{2, X}+\dot{\phi}^{2} f_{2, X X}\right)+\dot{\phi}^{2} f_{2, X \phi}\right\}-3 H \dot{\phi}^{2} n_{c}^{2} f_{2, n_{c} X}\right] \delta \phi+\frac{1}{a^{3}} \frac{\mathrm{d}}{\mathrm{d} t}\left\{a^{3}\left(\dot{\phi} D_{6}-2 H q_{t}\right) \alpha\right\} \\
& +\sum_{I=c, b, r}\left(\rho_{I}+P_{I}\right)\left(\dot{v}_{I}-3 H c_{I}^{2} v_{I}\right)+\left[f_{1}\left(\rho_{c}+P_{c}\right)-\dot{\phi} n_{c}^{2} f_{2, n_{c}}\right] \dot{v}_{c}-\left[\left\{3 H c_{c}^{2} f_{1}-\dot{\phi}\left(f_{1, \phi}+\ddot{\phi} f_{1, X}\right)\right\}\left(\rho_{c}+P_{c}\right)\right. \\
& \left.+n_{c}^{2}\left\{(\ddot{\phi}-3 H \dot{\phi}) f_{2, n_{c}}+\dot{\phi}^{2}\left(f_{2, n_{c} \phi}+\ddot{\phi} f_{2, n_{c} X}\right)\right\}-3 H \dot{\phi} n_{c}^{3} f_{2, n_{c} n_{c}}\right] v_{c}=0 .
\end{aligned}
$$

These equations can be used to eliminate $\alpha, \chi, v_{I}$, and $E$ from the action (3.37). In Sec. IVB, we will derive the stability conditions of dynamical perturbations in the small-scale limit after the elimination of nondynamical perturbations.

Variations of the action (3.37) with respect to dynamical fields $\delta \phi, \delta \rho_{I}$, and $\zeta$ lead to

$$
\begin{aligned}
& \dot{\mathcal{Z}}+3 H \mathcal{Z}+3\left(D_{7}-\dot{\phi} f_{1, X} \rho_{c}+\dot{\phi}^{2} n_{c} f_{2, X}\right) \dot{\zeta}+M_{\phi}^{2} \delta \phi-\left[\left\{\ddot{\phi}\left(f_{1, X \phi}+\dot{\phi}^{2} f_{1, X X \phi}\right)-f_{1, \phi \phi}+\dot{\phi}^{2} f_{1, X \phi \phi}\right\} \rho_{c}\right. \\
& \left.-3 H P_{c} \dot{\phi} f_{1, X \phi}-\dot{\phi} n_{c}\left\{\ddot{\phi}\left(3 f_{2, X \phi}+\dot{\phi}^{2} f_{2, X X \phi}\right)+\dot{\phi}^{2} f_{2, X \phi \phi}\right\}+3 H n_{c}^{2}\left(f_{2, n_{c} \phi}+\dot{\phi}^{2} f_{2, n_{c} X \phi}\right)\right] \delta \phi \\
& +\left[f_{1, \phi}-\frac{n_{c}}{\rho_{c}+P_{c}}\left(3 H n_{c} f_{2, n_{c}}+\dot{\phi} n_{c} f_{2, n_{c} \phi}-\dot{\phi} \ddot{\phi} f_{2, X}\right)\right] \delta \rho_{c}-\left[D_{5}-\left(f_{1, \phi}-\dot{\phi}^{2} f_{1, X \phi}\right) \rho_{c}-\dot{\phi}^{3} n_{c} f_{2, X \phi}\right] \alpha \\
& -\frac{k^{2}}{a^{2}}\left[2 D_{2} \delta \phi-D_{6} \alpha-D_{7} \chi+B_{1} \zeta-a^{2} B_{4} E+\left(f_{1, X} \rho_{c}-\dot{\phi} n_{c} f_{2, X}\right)\left\{\delta \phi+\dot{\phi}\left(\chi-a^{2} \dot{E}\right)\right\}\right]=0 \\
& \left(1+f_{1}-\frac{\dot{\phi} n_{c}^{2} f_{2, n_{c}}}{\rho_{c}+P_{c}}\right) \dot{v}_{c}-\left[3 H c_{c}^{2}\left(1+f_{1}\right)-\dot{\phi}\left(f_{1, \phi}+\ddot{\phi} f_{1, X}\right)+\frac{n_{c}^{2}}{\rho_{c}+P_{c}}\left\{\ddot{\phi} f_{2, n_{c}}-3 H \dot{\phi}\left(f_{2, n_{c}}+n_{c} f_{\left.2, n_{c} n_{c}\right)}\right)\right.\right. \\
& \left.\left.+\dot{\phi}^{2}\left(f_{2, n_{c} \phi}+\ddot{\phi} f_{2, n_{c} X}\right)\right\}\right] v_{c}-\left[1+f_{1}-\dot{\phi}^{2} f_{1, X}+\frac{\dot{\phi}^{3} n_{c}\left(f_{2, X}+n_{c} f_{2, n_{c} X}\right)}{\rho_{c}+P_{c}}\right] \alpha \\
& -\left[\dot{\phi} f_{1, X}-\frac{n_{c}\left(n_{c} f_{2, n_{c}}+\dot{\phi}^{2} f_{2, X}+\dot{\phi}^{2} n_{c} f_{2, n_{c} X}\right)}{\rho_{c}+P_{c}}\right] \dot{\delta \phi}-\left[f_{1, \phi}-\frac{n_{c}\left(3 H n_{c} f_{2, n_{c}}+\dot{\phi} n_{c} f_{2, n_{c} \phi}-\dot{\phi} \ddot{\phi} f_{2, X}\right)}{\rho_{c}}\right] \delta \phi \\
& -\frac{1}{\rho_{c}+P_{c}}\left[\left(1+f_{1}\right) c_{c}^{2}-\frac{\dot{\phi} n_{c}^{2}\left(2 f_{2, n_{c}}+n_{c} f_{2, n_{c} n_{c}}\right)}{\rho_{c}+P_{c}}\right] \delta \rho_{c}=0, \\
& \dot{v}_{I}-3 H c_{I}^{2} v_{I}-\frac{c_{I}^{2}}{\rho_{I}+P_{I}} \delta \rho_{I}-\alpha=0, \quad f_{0} \quad I=b, r \\
& \dot{\mathcal{W}}+3 H \mathcal{W}+\sum_{I=c, b, r}\left(\rho_{I}+P_{I}\right)\left(\dot{v}_{I}-3 H c_{I}^{2} v_{I}\right)+\left[\left(\rho_{c}+P_{c}\right) f_{1}-\dot{\phi} n_{c}^{2} f_{2, n_{c}}\right] \dot{v}_{c}+\frac{k^{2}}{3 a^{2}}\left(2 q_{t} \alpha+2 q_{t} \zeta+B_{1} \delta \phi\right) \\
& -\left[n _ { c } ^ { 2 } \left\{\ddot{\phi} f_{2, n_{c}}-3 H \dot{\phi}\left(f_{2, n_{c}}+n_{c} f_{2, n_{c} n_{c}}\right)+\dot{\phi}^{2}\left(f_{2, n_{c} \phi}+\ddot{\phi} f_{\left.\left.2, n_{c} X\right)\right\}}\right.\right.\right. \\
& \left.+\left(\rho_{c}+P_{c}\right)\left\{3 H c_{c}^{2} f_{1}-\dot{\phi}\left(f_{1, \phi}+\ddot{\phi} f_{1, X}\right)\right\}\right] v_{c}=0
\end{aligned}
$$

where

$$
M_{\phi}^{2} \equiv-2 D_{3},
$$




$$
\begin{aligned}
\mathcal{Z} \equiv & {\left[2 D_{1}-\left(f_{1, X}+\dot{\phi}^{2} f_{1, X X}\right) \rho_{c}+\dot{\phi} n_{c}\left(3 f_{2, X}+\dot{\phi}^{2} f_{2, X X}\right)\right] \dot{\delta \phi}+3 D_{6} \dot{\zeta}+\left[D_{4}+\dot{\phi}\left(f_{1, X}+\dot{\phi}^{2} f_{1, X X}\right) \rho_{c}\right.} \\
& \left.-\dot{\phi}^{2} n_{c}\left(3 f_{2, X}+\dot{\phi}^{2} f_{2, X X}\right)\right] \alpha-\left[\dot{\phi} f_{1, X}-\frac{n_{c}\left(n_{c} f_{2, n_{c}}+\dot{\phi}^{2} f_{2, X}+\dot{\phi}^{2} n_{c} f_{2, n_{c} X}\right)}{\rho_{c}+P_{c}}\right] \delta \rho_{c} \\
& +\frac{k^{2}}{a^{2}}\left[D_{6} \chi-a^{2}\left(D_{6} \dot{E}+D_{7} E\right)\right], \\
\mathcal{W} \equiv & 2 q_{t} \dot{\zeta}-D_{6} \dot{\delta \phi}+\left(D_{7}-\dot{\phi} f_{1, X} \rho_{c}+\dot{\phi}^{2} n_{c} f_{2, X}\right) \delta \phi+\left(\dot{\phi} D_{6}-2 H q_{t}\right) \alpha+\frac{2 k^{2}}{3 a^{2}} q_{t}\left(\chi-a^{2} \dot{E}\right) .
\end{aligned}
$$

The coefficient $-2 D_{3}$ contains the term $-G_{2, \phi \phi}$. For a canonical scalar with the potential $V(\phi)$, i.e., $G_{2}=X-V(\phi)$, the term $-G_{2, \phi \phi}$ reduces to $V_{, \phi \phi}$, which corresponds to the field mass squared. In Ref. [50], the scalar perturbation equations were derived in coupled quintessence with the interacting Lagrangian $\mathcal{L}_{\text {int }}=q J_{c}^{\mu} \partial_{\mu} \phi / n_{c}$, where $q$ is a constant. They can be recovered by setting $f_{1}=0, f_{2}=q / n_{c}, G_{2}=X-V, G_{3}=0, G_{4}=M_{\mathrm{pl}}^{2} / 2, \rho_{b}=0$, and $\rho_{r}=0$ in Eqs. (3.40)-(3.42), (3.44), (3.45), and (3.47).

On using Eqs. (3.43) and (3.47), the time derivatives $\ddot{\zeta}$ and $\ddot{\delta \phi}$ are eliminated to give

$$
q_{t}\left[\alpha+\dot{\chi}+\zeta+H \chi-a^{2}(\ddot{E}+3 H \dot{E})\right]+\dot{q}_{t}\left(\chi-a^{2} \dot{E}+\frac{\delta \phi}{\dot{\phi}}\right)=0,
$$

where we used the relation $B_{1}=2 \dot{q}_{t} / \dot{\phi}$. The above perturbation equations of motion can be applied to any choices of gauges.

\section{SMALL-SCALE STABILITY CONDITIONS}

By using the second-order action of scalar perturbations derived in Sec. III, we identify conditions for the absence of ghosts and Laplacian instabilities in the small-scale limit. Before doing so, we introduce commonly used gaugeinvariant variables and discuss several different choices of gauges.

\section{A. Gauge-invariant variables and gauge fixings}

Let us consider the infinitesimal gauge transformation of time and spatial coordinates of the forms $\tilde{t}=t+\xi^{0}$ and $\tilde{x}^{i}=x^{i}+\delta^{i j} \partial_{j} \xi$, where $\xi^{0}$ and $\xi$ are scalar quantities. Then, the four scalar metric perturbations in the line element (3.1) transform as [78-80]

$$
\tilde{\alpha}=\alpha-\dot{\xi}^{0}, \quad \tilde{\chi}=\chi+\xi^{0}-a^{2} \dot{\xi}, \quad \tilde{\zeta}=\zeta-H \xi^{0}, \quad \tilde{E}=E-\xi .
$$

The transformations of $\delta \phi, \delta \rho_{I}$, and $v_{I}$ are given, respectively, by

$$
\widetilde{\delta \phi}=\delta \phi-\dot{\phi} \xi^{0}, \quad \widetilde{\delta \rho_{I}}=\delta \rho_{I}-\dot{\rho}_{I} \xi^{0}, \quad \tilde{v}_{I}=v_{I}-\xi^{0} .
$$

Then, the following perturbed quantities are invariant under the gauge transformation,

$$
\begin{aligned}
& \delta \phi_{\mathrm{f}}=\delta \phi-\frac{\dot{\phi}}{H} \zeta, \quad \delta \rho_{I \mathrm{f}}=\delta \rho_{I}-\frac{\dot{\rho}_{I}}{H} \zeta, \quad v_{I \mathrm{f}}=v_{I}-\frac{\zeta}{H}, \\
& \mathcal{R}=\zeta-\frac{H}{\dot{\phi}} \delta \phi, \quad \delta \rho_{I \mathrm{u}}=\delta \rho_{I}-\frac{\dot{\rho}_{I}}{\dot{\phi}} \delta \phi, \quad v_{I \mathrm{u}}=v_{I}-\frac{\delta \rho_{I}}{\dot{\rho}_{I}}, \\
& \delta \phi_{\mathrm{N}}=\delta \phi+\dot{\phi}\left(\chi-a^{2} \dot{E}\right), \quad \delta \rho_{I \mathrm{~N}}=\delta \rho_{I}+\dot{\rho}_{I}\left(\chi-a^{2} \dot{E}\right), \quad v_{I \mathrm{~N}}=v_{I}+\chi-a^{2} \dot{E} .
\end{aligned}
$$

The gauge-invariant gravitational potentials first introduced by Bardeen [78] are given by

$$
\Psi=\alpha+\frac{\mathrm{d}}{\mathrm{d} t}\left(\chi-a^{2} \dot{E}\right), \quad \Phi=\zeta+H\left(\chi-a^{2} \dot{E}\right) .
$$

We recall that the background CDM density containing the effect of interactions is given by $\hat{\rho}_{c}=\left(1+f_{1}\right) \rho_{c}$. We also introduce the corresponding gauge-invariant CDM density perturbation, as

$$
\widehat{\delta \rho_{c \mathrm{~N}}}=\left(1+f_{1}\right) \delta \rho_{c \mathrm{~N}}+\left[f_{1, \phi} \delta \phi_{\mathrm{N}}+f_{1, X} \dot{\phi}\left(\dot{\delta} \phi_{\mathrm{N}}-\dot{\phi} \Psi\right)\right] \rho_{c}
$$


The gauge choice corresponds to fixing the infinitesimal scalars $\xi^{0}$ and $\xi$. The latter can be fixed by choosing

$$
E=0 .
$$

There are several different gauge choices for the fixing of $\xi^{0}$. The representative examples are

$$
\begin{array}{ll}
\text { (i) } \zeta=0 & \text { (Flat gauge) }, \\
\text { (ii) } \delta \phi=0 & \text { (Unitary gauge) }, \\
\text { (iii) } \chi=0 & \text { (Newtonian gauge) }
\end{array}
$$

In the flat gauge, the dynamical scalar perturbations are given by $\delta \phi_{\mathrm{f}}=\delta \phi$ and $\delta \rho_{I \mathrm{f}}=\delta \rho_{I}$, while, in the unitary gauge, they correspond to $\mathcal{R}=\zeta$ and $\delta \rho_{I \mathrm{u}}=\delta \rho_{I}$. We note that the comoving curvature perturbation $\mathcal{R}$ [81, 82] is related to the Mukhanov-Sasaki variable $\delta \phi_{\mathrm{f}}[83,84]$, as $\mathcal{R}=-(H / \dot{\phi}) \delta \phi_{\mathrm{f}}$. In the Newtonian gauge, the perturbations in Eqs. (4.5) and (4.6) reduce, respectively, to $\delta \phi_{\mathrm{N}}=\delta \phi, \delta \rho_{I \mathrm{~N}}=\delta \rho_{I}, v_{I \mathrm{~N}}=v_{I}, \Psi=\alpha$, and $\Phi=\zeta$. For this gauge choice, the gauge-invariant dynamical scalar perturbations are $\mathcal{R}=\Phi-(H / \dot{\phi}) \delta \phi_{\mathrm{N}}\left(\right.$ or $\left.\delta \phi_{\mathrm{f}}=\delta \phi_{\mathrm{N}}-(\dot{\phi} / H) \Phi\right)$ and $\delta \rho_{I \mathrm{~N}}$.

\section{B. Stability conditions in the small-scale limit}

In order to obtain conditions for the absence of ghosts and Laplacian instabilities, we choose two different gauges and show that the small-scale stability conditions are independent of the choice of gauges.

\section{Flat gauge}

Let us begin with the flat gauge characterized by $\zeta=0$ and $E=0$. We first solve Eqs. (3.40)-(3.42) for nondynamical perturbations $\alpha, \chi, v_{c}, v_{b}, v_{r}$ and substitute them into Eq. (3.37). After the integration by parts, the resulting secondorder action is expressed in the form

$$
\mathcal{S}_{s}^{(2)}=\int \mathrm{d} t \mathrm{~d}^{3} x a^{3}\left(\dot{\overrightarrow{\mathcal{X}}}^{t} \boldsymbol{K} \dot{\overrightarrow{\mathcal{X}}}-\frac{k^{2}}{a^{2}} \overrightarrow{\mathcal{X}}^{t} \boldsymbol{G} \overrightarrow{\mathcal{X}}-\overrightarrow{\mathcal{X}}^{t} \boldsymbol{M} \overrightarrow{\mathcal{X}}-\frac{k}{a} \overrightarrow{\mathcal{X}}^{t} \boldsymbol{B} \dot{\overrightarrow{\mathcal{X}}}\right)
$$

where $\boldsymbol{K}, \boldsymbol{G}, \boldsymbol{M}, \boldsymbol{B}$ are $4 \times 4$ matrices, and

$$
\overrightarrow{\mathcal{X}}^{t}=\left(\delta \phi_{\mathrm{f}}, \delta \rho_{c \mathrm{f}} / k, \delta \rho_{b \mathrm{f}} / k, \delta \rho_{r \mathrm{f}} / k\right) .
$$

The leading-order terms in the components of matrix $\boldsymbol{M}$ are of order $k^{0}$. Taking the small-scale limit $(k \rightarrow \infty)$, the nonvanishing matrix components of $\boldsymbol{K}$ and $\boldsymbol{G}$ are given by

$$
\begin{aligned}
K_{11}^{(\mathrm{f})}= & \frac{H^{2} q_{t} q_{s}}{\left(2 H q_{t}-\dot{\phi} D_{6}\right)^{2}}, \quad K_{22}^{(\mathrm{f})}=\frac{a^{2}}{2\left(\rho_{c}+P_{c}\right)}\left(1+f_{1}-\frac{\dot{\phi} n_{c}^{2} f_{2, n_{c}}}{\rho_{c}+P_{c}}\right) \\
K_{33}^{(\mathrm{f})}= & \frac{a^{2}}{2\left(\rho_{b}+P_{b}\right)}, \quad K_{44}^{(\mathrm{f})}=\frac{a^{2}}{2\left(\rho_{r}+P_{r}\right)}, \\
G_{11}^{(\mathrm{f})}= & -D_{2}+\frac{D_{6} D_{7}-\left[\sum_{I=c, b, r}\left(\rho_{I}+P_{I}\right)+f_{1}\left(\rho_{c}+P_{c}\right)-\dot{\phi} n_{c}^{2} f_{2, n_{c}}\right] \mathcal{G}_{1}}{2 H q_{t}-\dot{\phi} D_{6}}+\dot{\mathcal{G}}_{1}+H \mathcal{G}_{1} \\
& -\frac{\left(f_{1, X} \rho_{c}-\dot{\phi} n_{c} f_{2, X}\right)\left(2 H q_{t}+\dot{\phi} D_{6}\right)}{2\left(2 H q_{t}-\dot{\phi} D_{6}\right)}, \\
G_{22}^{(\mathrm{f})}= & \frac{a^{2}}{2\left(\rho_{c}+P_{c}\right)}\left[\left(1+f_{1}\right) c_{c}^{2}-\frac{\dot{\phi} n_{c}^{2}\left(2 f_{2, n_{c}}+n_{c} f_{2, n_{c} n_{c}}\right)}{\rho_{c}+P_{c}}\right], \quad G_{33}^{(\mathrm{f})}=\frac{a^{2} c_{b}^{2}}{2\left(\rho_{b}+P_{b}\right)}, \quad G_{44}^{(\mathrm{f})}=\frac{a^{2} c_{r}^{2}}{2\left(\rho_{r}+P_{r}\right)},(4)
\end{aligned}
$$

where

$$
\mathcal{G}_{1}=\frac{D_{6}^{2}}{2\left(2 H q_{t}-\dot{\phi} D_{6}\right)},
$$


and we used the relation (3.25). The leading-order contributions to the anti-symmetric matrix $\boldsymbol{B}$ are ${ }^{3}$

$$
B_{12}^{(\mathrm{f})}=-B_{21}^{(\mathrm{f})}=-\frac{a H q_{t}\left[\dot{\phi} f_{1, X}\left(\rho_{c}+P_{c}\right)-\dot{\phi}^{2} n_{c}\left(f_{2, X}+n_{c} f_{2, n_{c} X}\right)-n_{c}^{2} f_{2, n_{c}}\right]}{\left(2 H q_{t}-\dot{\phi} D_{6}\right)\left(\rho_{c}+P_{c}\right)},
$$

while the other components of $\boldsymbol{B}$ are lower than the order $k^{0}$.

We recall that $q_{t}$ and $q_{s}$ are defined, respectively, by Eqs. (2.26) and (2.31). The scalar ghost associated with the field perturbation $\delta \phi_{\mathrm{f}}$ is absent for $K_{11}^{(\mathrm{f})}>0$. Provided that there is no ghost in the tensor sector $\left(q_{t}>0\right)$, the condition $K_{11}^{(\mathrm{f})}>0$ translates to

$$
q_{s}>0 \text {. }
$$

Since $q_{s}$ contains the $X$ derivatives of $f_{1}$ and $f_{2}$, the $X$ dependence in $f_{1}$ and $f_{2}$ affects the no-ghost condition of $\delta \phi_{\mathrm{f}}$.

For the CDM perturbation $\delta \rho_{c f}$, the ghost is absent if $K_{22}^{(\mathrm{f})}>0$, i.e.,

$$
q_{c} \equiv 1+f_{1}-\frac{\dot{\phi} n_{c}^{2} f_{2, n_{c}}}{\rho_{c}+P_{c}}>0 .
$$

Hence the coupling $f_{1}$ and the $n_{c}$ dependence in $f_{2}$ lead to the modification to $q_{c}$. For baryons and radiations, the positivities of $K_{33}^{(\mathrm{f})}$ and $K_{44}^{(\mathrm{f})}$ are ensured under the weak energy conditions $(2.17)$.

Let us proceed to the discussion of the propagation speeds of perturbations (4.13), which are associated with conditions for the absence of Laplacian instabilities. We need to caution that the nonvanishing components $B_{12}^{(\mathrm{f})}$ and $B_{21}^{(\mathrm{f})}$ in Eq. (4.17) affect the propagation speeds of the perturbations $\mathcal{X}_{1} \equiv \delta \phi_{\mathrm{f}}$ and $\mathcal{X}_{2} \equiv \delta \rho_{c f} / k$. In the small-scale limit, the equations of motion for $\mathcal{X}_{1}$ and $\mathcal{X}_{2}$ following from the Lagrangian (4.12) are of the forms,

$$
\begin{aligned}
& K_{11}^{(\mathrm{f})} \ddot{\mathcal{X}}_{1}+\frac{k^{2}}{a^{2}} G_{11}^{(\mathrm{f})} \mathcal{X}_{1}+\frac{k}{a} B_{12}^{(\mathrm{f})} \dot{\mathcal{X}}_{2} \simeq 0 \\
& K_{22}^{(\mathrm{f})} \ddot{\mathcal{X}}_{2}+\frac{k^{2}}{a^{2}} G_{22}^{(\mathrm{f})} \mathcal{X}_{2}+\frac{k}{a} B_{21}^{(\mathrm{f})} \dot{\mathcal{X}}_{1} \simeq 0
\end{aligned}
$$

where we neglected the terms lower than the order of $\omega^{2}, \omega k$, and $k^{2}$ in the dispersion relation ( $\omega$ is a frequency). Substituting the solutions $\mathcal{X}_{i}=\tilde{\mathcal{X}}_{i} e^{i(\omega t-k x)}$ with $i=1,2$ into Eqs. (4.20)-(4.21), where $\tilde{\mathcal{X}}_{i}$ are constants, it follows that

$$
\begin{aligned}
& \omega^{2} \tilde{\mathcal{X}}_{1}-\hat{c}_{s}^{2} \frac{k^{2}}{a^{2}} \tilde{\mathcal{X}}_{1}-i \omega \frac{k}{a} \frac{B_{12}^{(\mathrm{f})}}{K_{11}^{(\mathrm{f})}} \tilde{\mathcal{X}}_{2}=0, \\
& \omega^{2} \tilde{\mathcal{X}}_{2}-\hat{c}_{c}^{2} \frac{k^{2}}{a^{2}} \tilde{\mathcal{X}}_{2}-i \omega \frac{k}{a} \frac{B_{21}^{(\mathrm{f})}}{K_{22}^{(\mathrm{f})}} \tilde{\mathcal{X}}_{1}=0,
\end{aligned}
$$

where

$$
\begin{aligned}
& \hat{c}_{s}^{2}=\frac{G_{11}^{(\mathrm{f})}}{K_{11}^{(\mathrm{f})}} \\
& \hat{c}_{c}^{2}=\frac{G_{22}^{(\mathrm{f})}}{K_{22}^{(\mathrm{f})}}=c_{c}^{2}-\frac{\left[\left(2-c_{c}^{2}\right) f_{2, n_{c}}+n_{c} f_{2, n_{c} n_{c}}\right] \dot{\phi} n_{c}^{2}}{\left(1+f_{1}\right)\left(\rho_{c}+P_{c}\right)-\dot{\phi} n_{c}^{2} f_{2, n_{c}}} .
\end{aligned}
$$

If the coupling $f$ is absent, we have $B_{12}^{(\mathrm{f})}=-B_{21}^{(\mathrm{f})}=0$ and hence the propagation speeds of $\mathcal{X}_{1}$ and $\mathcal{X}_{2}$ reduce to those given in Eqs. (4.24) and (4.25), respectively. The interaction between these two fields gives rise to the mixing terms in Eqs. (4.22) and (4.23). In the following, we consider the interacting theories obeying the condition

$$
\hat{c}_{c}^{2}=0
$$

\footnotetext{
${ }^{3}$ After this paper was published in Phys. Rev. D 101, 063511 (2020), we noticed that the propagation speed squared of the scalar field $c_{s}^{2}$ is affected by the off-diagonal components of $\boldsymbol{B}$. The value of $\hat{c}_{s}^{2}$ defined in Eq. (4.24) is identical to $c_{s}^{2}$ used in the published version. As we explicitly show in this arXiv version, the off-diagonal component $B_{12}^{(\mathrm{f})}$ leads to the modification $\Delta c_{s}^{2}=\left(B_{12}^{(\mathrm{f})}\right)^{2} /\left(K_{11}^{(\mathrm{f})} K_{22}^{(\mathrm{f})}\right)$ to $\hat{c}_{s}^{2}$, where $\Delta c_{s}^{2}$ is positive as long as the no-ghost conditions (4.18) and (4.19) are satisfied.
} 
For CDM satisfying $c_{c}^{2}=0$, Eq. (4.25) shows that the coupling $f_{2}$ obeys the relation $2 f_{2, n_{c}}+n_{c} f_{2, n_{c} n_{c}}=0$, i.e.,

$$
f_{2} \propto n_{c}^{-1} .
$$

Substituting Eq. (4.26) into Eq. (4.23), it follows that

$$
\begin{aligned}
& \omega=0, \\
& \omega \tilde{\mathcal{X}}_{2}-i \frac{k}{a} \frac{B_{21}^{(\mathrm{f})}}{K_{22}^{(\mathrm{f})}} \tilde{\mathcal{X}}_{1}=0 .
\end{aligned}
$$

The solution (4.28) corresponds to the dispersion relation for CDM perturbations, so that the CDM propagation speed squared $c_{\mathrm{CDM}}^{2}$ is identical to 0 . Then, there is no additional pressure affecting the growth of CDM density perturbations. Substituting the other solution (4.29) into Eq. (4.22), we obtain the dispersion relation $\omega^{2}=c_{s}^{2} k^{2} / a^{2}$ for the scalar perturbation $\delta \phi_{\mathrm{f}}$, with

$$
c_{s}^{2}=\hat{c}_{s}^{2}+\Delta c_{s}^{2}
$$

where $\hat{c}_{s}^{2}$ is given by Eq. (4.24), and

$$
\Delta c_{s}^{2}=\frac{\left(B_{12}^{(\mathrm{f})}\right)^{2}}{K_{11}^{(\mathrm{f})} K_{22}^{(\mathrm{f})}} .
$$

The Laplacian instability is absent for

$$
c_{s}^{2} \geq 0
$$

Under the no-ghost conditions $K_{11}^{(\mathrm{f})}>0$ and $K_{22}^{(\mathrm{f})}>0$, the correction term $\Delta c_{s}^{2}$ arising from the off-diagonal components of $\boldsymbol{B}$ is always positive. This means that, as long as $\hat{c}_{s}^{2} \geq 0$, the condition (4.32) always holds.

The propagation speeds of baryon and radiation density perturbations are not affected by the off-diagonal components of $\boldsymbol{B}$, so they are given, respectively, by $c_{b}^{2}=G_{33}^{(\mathrm{f})} / K_{33}^{(\mathrm{f})}$ and $c_{r}^{2}=G_{44}^{(\mathrm{f})} / K_{44}^{(\mathrm{f})}$, both of which should be non-negative to avoid Laplacian instabilities.

\section{Unitary gauge}

We also derive stability conditions of scalar perturbations by choosing the unitary gauge characterized by $\delta \phi=0$ and $E=0$. After eliminating the nondynamical perturbations $\alpha, \chi, v_{c}, v_{b}, v_{r}$ on account of Eqs. (3.40)-(3.42), the second-order scalar action (3.37) reduces to the form (4.12), with the dynamical perturbations given by

$$
\overrightarrow{\mathcal{X}}^{t}=\left(\mathcal{R}, \delta \rho_{c \mathrm{u}} / k, \delta \rho_{b \mathrm{u}} / k, \delta \rho_{r \mathrm{u}} / k\right) .
$$

In the small-scale limit, the nonvanishing components of $\boldsymbol{K}$ and $\boldsymbol{G}$ are again diagonal terms, which are given by

$$
\begin{aligned}
& K_{11}^{(\mathrm{u})}=\frac{\dot{\phi}^{2} q_{t} q_{s}}{\left(2 H q_{t}-\dot{\phi} D_{6}\right)^{2}}, \quad K_{22}^{(\mathrm{u})}=K_{22}^{(\mathrm{f})}, \quad K_{33}^{(\mathrm{u})}=K_{33}^{(\mathrm{f})}, \quad K_{44}^{(\mathrm{u})}=K_{44}^{(\mathrm{f})}, \\
& G_{11}^{(\mathrm{u})}=-q_{t}-\frac{\left[\sum_{I=c, b, r}\left(\rho_{I}+P_{I}\right)+f_{1}\left(\rho_{c}+P_{c}\right)-\dot{\phi} n_{c}^{2} f_{2, n_{c}}\right] \mathcal{G}_{2}}{2 H q_{t}-\dot{\phi} D_{6}}+\dot{\mathcal{G}}_{2}+H \mathcal{G}_{2}, \\
& G_{22}^{(\mathrm{u})}=G_{22}^{(\mathrm{f})}, \quad G_{33}^{(\mathrm{u})}=G_{33}^{(\mathrm{f})}, \quad G_{44}^{(\mathrm{u})}=G_{44}^{(\mathrm{f})},
\end{aligned}
$$

where

$$
\mathcal{G}_{2}=\frac{2 q_{t}^{2}}{2 H q_{t}-\dot{\phi} D_{6}}
$$

The leading-order terms to the matrix components of $\boldsymbol{B}$ are

$$
B_{12}^{(\mathrm{u})}=-B_{21}^{(\mathrm{u})}=\frac{a \dot{\phi} q_{t}\left[\dot{\phi} f_{1, X}\left(\rho_{c}+P_{c}\right)-\dot{\phi}^{2} n_{c}\left(f_{2, X}+n_{c} f_{2, n_{c} X}\right)-n_{c}^{2} f_{2, n_{c}}\right]}{\left(2 H q_{t}-\dot{\phi} D_{6}\right)\left(\rho_{c}+P_{c}\right)} .
$$


The stability conditions of baryon and radiation density perturbations are the same as those derived in the flat gauge. For the perturbations $\mathcal{R}$ and $\delta \rho_{c u} / k$, it is convenient to notice the following relations,

$$
\frac{K_{11}^{(\mathrm{u})}}{K_{11}^{(\mathrm{f})}}=\frac{\dot{\phi}^{2}}{H^{2}}, \quad \frac{K_{22}^{(\mathrm{u})}}{K_{22}^{(\mathrm{f})}}=1, \quad \frac{G_{11}^{(\mathrm{u})}}{G_{11}^{(\mathrm{f})}}=\frac{\dot{\phi}^{2}}{H^{2}}, \quad \frac{G_{22}^{(\mathrm{u})}}{G_{22}^{(\mathrm{f})}}=1, \quad \frac{B_{12}^{(\mathrm{u})}}{B_{12}^{(\mathrm{f})}}=-\frac{\dot{\phi}}{H},
$$

where we used Eqs. (2.20), (2.21), and (3.23) for the derivation of the third equality. This means that the no-ghost conditions $K_{11}^{(\mathrm{u})}>0$ and $K_{22}^{(\mathrm{u})}>0$ in the unitary gauge are identical to those obtained in the flat gauge.

For the interacting theories satisfying the condition $\hat{c}_{c}^{2}=G_{22}^{(\mathrm{u})} / K_{22}^{(\mathrm{u})}=G_{22}^{(\mathrm{f})} / K_{22}^{(\mathrm{f})}=0$, we obtain the same dispersion relation of CDM as Eq. (4.28), so that $c_{\mathrm{CDM}}^{2}$ vanishes. The propagation speed squared for $\mathcal{R}$ is given by Eq. (4.30), with

$$
\hat{c}_{s}^{2}=\frac{G_{11}^{(\mathrm{u})}}{K_{11}^{(\mathrm{u})}}=\frac{G_{11}^{(\mathrm{f})}}{K_{11}^{(\mathrm{f})}}, \quad \Delta c_{s}^{2}=\frac{\left(B_{12}^{(\mathrm{u})}\right)^{2}}{K_{11}^{(\mathrm{u})} K_{22}^{(\mathrm{u})}}=\frac{\left(B_{12}^{(\mathrm{f})}\right)^{2}}{K_{11}^{(\mathrm{f})} K_{22}^{(\mathrm{f})}},
$$

where we used Eq. (4.38). Thus, $c_{s}^{2}$ is equivalent to each other in both unitary and flat gauges.

The matrix component $G_{11}^{(\mathrm{u})}$ in Eq. (4.35) contains the time derivative of $\mathcal{G}_{2}$, which generates the terms $\dot{q}_{t}$, $\dot{D}_{6}$, and $\ddot{\phi}$ in $\hat{c}_{s}^{2}$. We eliminate these time derivatives by using Eqs. $(2.20),(2.21),(3.23)$, and the relation $B_{1}=2 \dot{q}_{t} / \dot{\phi}$. Moreover, it is possible to express $\Delta c_{s}^{2}$ in a compact form by using $q_{s}$ and $q_{c}$. Then, the propagation speed squared of the scalar field is given by $c_{s}^{2}=\hat{c}_{s}^{2}+\Delta c_{s}^{2}$, where

$$
\begin{aligned}
\hat{c}_{s}^{2} & =-\frac{D_{6}^{2}+2 B_{1} D_{6}+2 q_{t}\left(2 D_{2}+f_{1, X} \rho_{c}-\dot{\phi} n_{c} f_{2, X}\right)}{q_{s}}, \\
\Delta c_{s}^{2} & =\frac{2 q_{t}\left[\dot{\phi} f_{1, X}\left(\rho_{c}+P_{c}\right)-\dot{\phi}^{2} n_{c}\left(f_{2, X}+n_{c} f_{2, n_{c} X}\right)-n_{c}^{2} f_{2, n_{c}}\right]^{2}}{q_{s} q_{c}\left(\rho_{c}+P_{c}\right)} .
\end{aligned}
$$

The couplings $f_{1}$ and $f_{2}$ nontrivially modify the value of $c_{s}^{2}$ in comparison to uncoupled theories.

The above discussion shows that, in the small-scale limit, the conditions for the absence of ghosts and Laplacian instabilities are independent of the choice of gauges. Indeed, the analysis in the Newtonian gauge also leads to the same stability conditions as those derived above (as performed for the uncoupled case in Ref. [64]).

\section{EFFECTIVE GRAVITATIONAL COUPLINGS FOR NONRELATIVISTIC MATTER}

We proceed to the derivation of the effective gravitational couplings felt by CDM and baryons for perturbations deep inside the sound horizon. Since we are interested in the cosmological dynamics in the late Universe, we ignore the contribution of radiations to the background and perturbation equations of motion. For CDM and baryons, we consider nonrelativistic matter satisfying

$$
P_{I}=0, \quad c_{I}^{2}=0, \quad \text { for } I=c, b .
$$

Even under the conditions (5.1), the $n_{c}$ dependence in $f_{2}$ gives rise to the nonvanishing value of $\hat{c}_{c}^{2}$ for CDM while the effective pressure $\hat{P}_{c}$ vanishes. We are primarily interested in interacting theories with $\hat{c}_{c}^{2}=0$, but we will keep $\hat{c}_{c}^{2}$ for the derivation of effective gravitational couplings. From Eq. (4.5), we define the gauge-invariant matter density contrast,

$$
\delta_{I \mathrm{~N}} \equiv \frac{\delta \rho_{I \mathrm{~N}}}{\rho_{I}}=\frac{\delta \rho_{I}}{\rho_{I}}-3 H\left(\chi-a^{2} \dot{E}\right), \quad \text { for } \quad I=c, b,
$$

where we used the continuity Eq. (2.16). From Eq. (3.42), the matter density contrast obeys

$$
\dot{\delta}_{I \mathrm{~N}}+\frac{k^{2}}{a^{2}} v_{I \mathrm{~N}}+3 \dot{\Phi}=0
$$

where $v_{I \mathrm{~N}}$ and $\Phi$ are gauge-invariant quantities defined in Eqs. (4.5) and (4.6).

From Eqs. (3.45) and (3.46), the velocity potentials of CDM and baryons satisfy

$$
\begin{aligned}
& \dot{v}_{c \mathrm{~N}}+a_{1} H v_{c \mathrm{~N}}-\left(1+a_{2}\right) \Psi+\frac{H}{\dot{\phi}}\left[a_{2} \frac{\dot{\delta} \phi_{\mathrm{N}}}{H}-\left(3 \hat{c}_{c}^{2}+a_{1}+a_{2} \epsilon_{\phi}\right) \delta \phi_{\mathrm{N}}\right]-\hat{c}_{c}^{2} \delta_{c \mathrm{~N}}=0, \\
& \dot{v}_{b \mathrm{~N}}-\Psi=0
\end{aligned}
$$


respectively, where

$$
a_{1}=\frac{\dot{q}_{c}}{H q_{c}}, \quad a_{2}=-\frac{\dot{\phi}\left[\rho_{c} \dot{\phi} f_{1, X}-\dot{\phi}^{2} n_{c}\left(f_{2, X}+n_{c} f_{2, n_{c} X}\right)-n_{c}^{2} f_{2, n_{c}}\right]}{\left(1+f_{1}\right) \rho_{c}-\dot{\phi} n_{c}^{2} f_{2, n_{c}}}, \quad \epsilon_{\phi}=\frac{\ddot{\phi}}{H \dot{\phi}} .
$$

Taking the time derivatives of Eq. (5.3) for $I=c, b$ and using Eqs. (5.4) and (5.5), it follows that

$$
\begin{aligned}
& \ddot{\delta}_{c \mathrm{~N}}+\left(2+a_{1}\right) H \dot{\delta}_{c \mathrm{~N}}+\hat{c}_{c}^{2} \frac{k^{2}}{a^{2}} \delta_{c \mathrm{~N}}+\left(1+a_{2}\right) \frac{k^{2}}{a^{2}} \Psi-\frac{k^{2}}{a^{2}} \frac{H}{\dot{\phi}}\left[a_{2} \frac{\dot{\delta} \phi_{\mathrm{N}}}{H}-\left(3 \hat{c}_{c}^{2}+a_{1}+\epsilon_{\phi} a_{2}\right) \delta \phi_{\mathrm{N}}\right] \\
& =-3 \ddot{\Phi}-3\left(2+a_{1}\right) H \dot{\Phi}, \\
& \ddot{\delta}_{b \mathrm{~N}}+2 H \dot{\delta}_{b \mathrm{~N}}+\frac{k^{2}}{a^{2}} \Psi=-3 \ddot{\Phi}-6 H \dot{\Phi} .
\end{aligned}
$$

The scalar perturbation equations derived in Sec. III B can be expressed in terms of the gauge-invariant gravitational potentials $\Psi$ and $\Phi$ as well as the other gauge-invariant perturbations given in Eq. (4.5). First of all, Eq. (3.51) translates to

$$
\Psi+\Phi=-\frac{H \alpha_{\mathrm{M}}}{\dot{\phi}} \delta \phi_{\mathrm{N}}
$$

where

$$
\alpha_{\mathrm{M}}=\frac{\dot{q}_{t}}{H q_{t}}
$$

\section{A. Quasi-static approximation for the modes deep inside the sound horizon}

In what follows, we employ the so-called quasi-static approximation for the modes deep inside the sound horizon [85-87]. Under this approximation scheme, the dominant contributions to the scalar perturbation equations of motion are the terms containing $\delta_{c \mathrm{~N}}, \dot{\delta}_{c \mathrm{~N}}, \delta_{b \mathrm{~N}}$, and $k^{2} / a^{2}$. We also take into account the field mass squared $M_{\phi}^{2}$ appearing in Eq. (3.44) to accommodate the case in which the field is heavy at high redshifts as in $f(R)$ models of late-time cosmic acceleration [88-90]. The tachyonic instability can be avoided for $M_{\phi}^{2} \geq 0$. Applying the quasi-static approximation to Eqs. (3.40) and (3.44), it follows that

$$
\begin{aligned}
& \frac{k^{2}}{a^{2}}\left(2 q_{t} \Phi-D_{6} \delta \phi_{\mathrm{N}}\right)-\left(1+a_{2}\right) q_{c} \rho_{c} \delta_{c \mathrm{~N}}-\rho_{b} \delta_{b \mathrm{~N}} \simeq 0 \\
& M_{\phi}^{2} \delta \phi_{\mathrm{N}}+\frac{k^{2}}{a^{2}}\left[\frac{1}{2 q_{t}}\left(q_{s} \hat{c}_{s}^{2}+D_{6}^{2}+\frac{4 H q_{t} D_{6} \alpha_{\mathrm{M}}}{\dot{\phi}}\right) \delta \phi_{\mathrm{N}}+D_{6} \Psi-\frac{2 H \alpha_{\mathrm{M}} q_{t}}{\dot{\phi}} \Phi\right] \\
& +\frac{H q_{c} \rho_{c}}{\dot{\phi}}\left[a_{2} \frac{\dot{\delta}_{c \mathrm{~N}}}{H}+\left\{3 \hat{c}_{c}^{2}+a_{1}+a_{2}\left(a_{1}+\epsilon_{a_{2}}\right)\right\} \delta_{c \mathrm{~N}}\right] \simeq 0,
\end{aligned}
$$

where we used Eq. (4.40), and

$$
\epsilon_{a_{2}}=\frac{\dot{a}_{2}}{H a_{2}}
$$

The difference from the uncoupled Horndeski theories is that there exists the time derivative $\dot{\delta}_{c \mathrm{~N}}$ in Eq. (5.12). We solve Eqs. (5.9), (5.11), and (5.12) for $\Psi, \Phi$, and $\delta \phi_{\mathrm{N}}$ to express them in terms of $\delta_{c \mathrm{~N}}, \dot{\delta}_{c \mathrm{~N}}$, and $\delta_{b \mathrm{~N}}$. In doing so, we introduce the following dimensionless variables ${ }^{4}$,

$$
\begin{aligned}
& \Delta_{1}=\alpha_{\mathrm{B}}-\alpha_{\mathrm{M}}, \quad \Delta_{2}=\frac{\dot{\phi}^{2} q_{s} \hat{c}_{s}^{2}}{4 H^{2} q_{t}^{2}}\left(1+\frac{2 q_{t} a^{2} M_{\phi}^{2}}{q_{s} \hat{c}_{s}^{2} k^{2}}\right), \quad \Delta_{3}=\left(1+a_{2}\right) \Delta_{1}+3 \hat{c}_{c}^{2}+a_{1}+a_{2}\left(a_{1}+\epsilon_{a_{2}}\right), \\
& \alpha_{\mathrm{B}}=-\frac{D_{6} \dot{\phi}}{2 H q_{t}}
\end{aligned}
$$

\footnotetext{
4 The definition of $\Delta_{2}$ in Eq. (6.14) of Ref. [64] contains the term $q_{t}$ in the denominator, but the definition of $\Delta_{2}$ in Eq. (5.14) of this paper is different from the former in that $q_{t}$ is replaced by $q_{t}^{2}$ to make $\Delta_{2}$ dimensionless.
} 
Then, $\Psi, \Phi$, and $\delta \phi_{\mathrm{N}}$ are expressed in the forms,

$$
\begin{aligned}
\Psi & =-\frac{a^{2}}{2 q_{t} \Delta_{2} k^{2}}\left[\left\{\Delta_{1} \Delta_{3}+\left(1+a_{2}\right) \Delta_{2}\right\} q_{c} \rho_{c} \delta_{c \mathrm{~N}}+\left(\Delta_{1}^{2}+\Delta_{2}\right) \rho_{b} \delta_{b \mathrm{~N}}+a_{2} q_{c} \Delta_{1} \rho_{c} \frac{\dot{\delta}_{c \mathrm{~N}}}{H}\right], \\
\Phi & =\frac{a^{2}}{2 q_{t} \Delta_{2} k^{2}}\left[\left\{\alpha_{\mathrm{B}} \Delta_{3}+\left(1+a_{2}\right) \Delta_{2}\right\} q_{c} \rho_{c} \delta_{c \mathrm{~N}}+\left(\alpha_{\mathrm{B}} \Delta_{1}+\Delta_{2}\right) \rho_{b} \delta_{b \mathrm{~N}}+a_{2} q_{c} \alpha_{\mathrm{B}} \rho_{c} \frac{\dot{\delta}_{c \mathrm{~N}}}{H}\right], \\
\delta \phi_{\mathrm{N}} & =-\frac{a^{2} \dot{\phi}}{2 H q_{t} \Delta_{2} k^{2}}\left(\Delta_{3} q_{c} \rho_{c} \delta_{c \mathrm{~N}}+\Delta_{1} \rho_{b} \delta_{b \mathrm{~N}}+a_{2} q_{c} \rho_{c} \frac{\dot{\delta}_{c \mathrm{~N}}}{H}\right) .
\end{aligned}
$$

The gravitational slip parameter is given by

$$
\eta \equiv-\frac{\Phi}{\Psi}=\frac{\left[\alpha_{\mathrm{B}}\left(\Delta_{3}+a_{2} \epsilon_{\delta_{c}}\right)+\left(1+a_{2}\right) \Delta_{2}\right] q_{c} \Omega_{c} \delta_{c \mathrm{~N}}+\left(\alpha_{\mathrm{B}} \Delta_{1}+\Delta_{2}\right) \Omega_{b} \delta_{b \mathrm{~N}}}{\left[\Delta_{1}\left(\Delta_{3}+a_{2} \epsilon_{\delta_{c}}\right)+\left(1+a_{2}\right) \Delta_{2}\right] q_{c} \Omega_{c} \delta_{c \mathrm{~N}}+\left(\Delta_{1}^{2}+\Delta_{2}\right) \Omega_{b} \delta_{b \mathrm{~N}}}
$$

where

$$
\Omega_{c}=\frac{\rho_{c}}{3 M_{\mathrm{pl}}^{2} H^{2}}, \quad \Omega_{b}=\frac{\rho_{b}}{3 M_{\mathrm{pl}}^{2} H^{2}}, \quad \epsilon_{\delta_{c}}=\frac{\dot{\delta}_{c}}{H \delta_{c}} .
$$

We substitute Eqs. (5.15), (5.17), and the time derivative of Eq. (5.17) into Eqs. (5.7) and (5.8). In doing so, the terms on the right hand sides of Eqs. (5.7) and (5.8) are neglected relative to those on their left hand sides. We also introduce the following dimensionless variables,

$$
Q_{t}=\frac{q_{t}}{M_{\mathrm{pl}}^{2}}, \quad \epsilon_{H}=\frac{\dot{H}}{H^{2}}, \quad \epsilon_{\Delta_{i}}=\frac{\dot{\Delta}_{i}}{H \Delta_{i}} \quad(\text { for } i=1,2,3),
$$

and

$$
b_{1}=\frac{3 a_{2}^{2} q_{c} \Omega_{c}}{2 Q_{t} \Delta_{2}}, \quad b_{2}=-b_{1}\left(1+2 \epsilon_{H}+\alpha_{\mathrm{M}}+\epsilon_{\Delta_{2}}-2 a_{1}-2 \epsilon_{a_{2}}\right), \quad b_{3}=\frac{3 a_{2} \Delta_{1} \Omega_{b}}{4 Q_{t} \Delta_{2}},
$$

where $b_{1}>0$ under the absence of ghosts and Laplacian/tachyonic instabilities. Then, it follows that

$$
\begin{aligned}
& \ddot{\delta}_{c \mathrm{~N}}+\frac{2+a_{1}+b_{2}}{1+b_{1}} H \dot{\delta}_{c \mathrm{~N}}+\frac{2 b_{3}}{1+b_{1}} H \dot{\delta}_{b \mathrm{~N}}+\frac{\hat{c}_{c}^{2}}{1+b_{1}} \frac{k^{2}}{a^{2}} \delta_{c \mathrm{~N}}-\frac{3 H^{2}}{2 G}\left(G_{c c} \Omega_{c} \delta_{c \mathrm{~N}}+G_{c b} \Omega_{b} \delta_{b \mathrm{~N}}\right) \simeq 0 \\
& \ddot{\delta}_{b \mathrm{~N}}+2 H \dot{\delta}_{b \mathrm{~N}}-2 H q_{c} b_{3} \frac{\Omega_{c}}{\Omega_{b}} \dot{\delta}_{c \mathrm{~N}}-\frac{3 H^{2}}{2 G}\left(G_{b c} \Omega_{c} \delta_{c \mathrm{~N}}+G_{b b} \Omega_{b} \delta_{b \mathrm{~N}}\right) \simeq 0
\end{aligned}
$$

where $G=1 /\left(8 \pi M_{\mathrm{pl}}^{2}\right)$, and the effective gravitational couplings are given by

$$
\begin{aligned}
G_{c c} & =\frac{2 q_{c}}{2 Q_{t} \Delta_{2}+3 a_{2}^{2} q_{c} \Omega_{c}}\left[\left(1+a_{2}\right)^{2} \Delta_{2}+\Delta_{3}\left\{\Delta_{1}+3 \hat{c}_{c}^{2}+a_{1}+a_{2}\left(1+\epsilon_{H}+\alpha_{\mathrm{M}}+\Delta_{1}-a_{1}+\epsilon_{\Delta_{2}}-\epsilon_{\Delta_{3}}\right)\right\}\right] G \\
G_{c b} & =\frac{2}{2 Q_{t} \Delta_{2}+3 a_{2}^{2} q_{c} \Omega_{c}}\left[\left(1+a_{2}\right)\left(\Delta_{1}^{2}+\Delta_{2}\right)+\Delta_{1}\left\{3 \hat{c}_{c}^{2}+a_{1}+a_{2}\left(1+\alpha_{\mathrm{M}}+\epsilon_{H}-\epsilon_{\Delta_{1}}+\epsilon_{\Delta_{2}}\right)\right\}\right] G \\
G_{b c} & =\frac{q_{c}\left[\Delta_{1} \Delta_{3}+\left(1+a_{2}\right) \Delta_{2}\right]}{Q_{t} \Delta_{2}} G \\
G_{b b} & =\frac{\Delta_{1}^{2}+\Delta_{2}}{Q_{t} \Delta_{2}} G
\end{aligned}
$$

The gravitational couplings $G_{c c}$ and $G_{c b}$, which are multiplied by $\Omega_{c} \delta_{c \mathrm{~N}}$ and $\Omega_{b} \delta_{b \mathrm{~N}}$ respectively, affect the growth of CDM density contrast $\delta_{c \mathrm{~N}}$. Meanwhile, the baryon density contrast $\delta_{b \mathrm{~N}}$ has the gravitational couplings multiplied by $\Omega_{b} \delta_{b \mathrm{~N}}$ and $\Omega_{c} \delta_{c \mathrm{~N}}$, respectively. The baryon perturbation is affected by the evolution of the CDM perturbation, and vice versa.

In the absence of matter couplings $\left(f_{1}=0=f_{2}\right)$, the quantities $a_{1}, a_{2}, b_{1}, b_{2}, b_{3}$ vanish. In this case we have $q_{c}=1$, $\hat{c}_{c}^{2}=0$ and $\Delta_{3}=\Delta_{1}$, so that $G_{c c}, G_{c b}, G_{b c}$ reduce to the same form as $G_{b b}$. We note that $G_{b b}$ is equivalent to the effective gravitational coupling $G_{\text {eff }}$ derived for uncoupled Horndeski theories [64, 87]. When the matter couplings $f_{1}$ and $f_{2}$ are present, $G_{c c}, G_{c b}, G_{b c}$ are generally different from $G_{b b}$. 
Apart from the specific coupling having the dependence $f_{2} \propto n_{c}^{-1}, \hat{c}_{c}^{2}$ is different from 0 . For $\hat{c}_{c}^{2}>0$, the growth of $\delta_{c \mathrm{~N}}$ is prevented by the positive pressure induced by the coupling $f_{2}\left(n_{c}\right)$, whereas, for $\hat{c}_{c}^{2}<0$, there is the additional enhancement of $\delta_{c \mathrm{~N}}$ besides the growth induced by gravitational instabilities. For the consistency with the observed galaxy power spectrum in the linear regime of perturbations, the effective sound speed squared needs to be much smaller than 1 , say, $\left|\hat{c}_{c}^{2}\right| \lesssim 10^{-5}$ in unified perfect fluid models of dark energy and dark matter [91]. We note that the $\phi$ and $X$ dependence in $f_{1}$ and $f_{2}$ does not modify the value of $\hat{c}_{c}^{2}$.

The above results have been obtained for the gauge-invariant density contrasts (5.2) for CDM and baryons. There is also the gauge-invariant CDM density perturbation (4.7) containing the effect of coupling $f_{1}$. Dividing $\widehat{\delta \rho_{c \mathrm{~N}}}$ by the background total CDM density $\hat{\rho}_{c}=\left(1+f_{1}\right) \rho_{c}$, the corresponding density contrast is

$$
\hat{\delta}_{c \mathrm{~N}} \equiv \frac{\widehat{\delta \rho_{c \mathrm{~N}}}}{\hat{\rho}_{c}}=\delta_{c \mathrm{~N}}+\frac{f_{1, \phi} \delta \phi_{\mathrm{N}}+f_{1, X} \dot{\phi}\left(\dot{\delta} \phi_{\mathrm{N}}-\dot{\phi} \Psi\right)}{1+f_{1}}
$$

Under the quasi-static approximation, the terms containing $\delta \phi_{\mathrm{N}}, \dot{\delta} \phi_{\mathrm{N}}$, and $\Psi$ in Eq. (5.28) are suppressed relative to $\delta_{c \mathrm{~N}}$. Indeed, this property can be confirmed by the solutions (5.15) and (5.17) in the small-scale limit. In this case, we have $\hat{\delta}_{c \mathrm{~N}} \simeq \delta_{c \mathrm{~N}}$ and hence the effective gravitational couplings for $\hat{\delta}_{c \mathrm{~N}}$ are approximately the same as those derived above for the perturbations deep inside the sound horizon.

\section{B. Concrete theories}

On using the general formulas (5.24)-(5.27), we compute the effective gravitational couplings for specific theories and the choice of couplings. We consider three different cases: (i) $f_{1}=f_{1}(\phi), f_{2}=0$, (ii) $f_{1}=f_{1}(\phi), f_{2}=f_{2}\left(n_{c}, \phi\right)$, and (iii) $f_{1}=0, f_{2}=f_{2}\left(n_{c}, X\right)$.

\section{Theories with $f_{1}=f_{1}(\phi)$ and $f_{2}=0$}

In this case, we introduce the dimensionless coupling

$$
Q=\frac{M_{\mathrm{pl}} f_{1, \phi}}{1+f_{1}} .
$$

Then, the right hand sides of Eqs. (2.35) and (2.40) reduce to $Q \hat{\rho}_{c} \dot{\phi} / M_{\mathrm{pl}}$ and $-Q \hat{\rho}_{c} \dot{\phi} / M_{\mathrm{pl}}$, respectively, showing that the quantity $Q$ characterizes the strength of nonminimal coupling $f_{1}(\phi)$. The quantities $a_{1}, a_{2}, q_{c}, \hat{c}_{c}^{2}$, and $\Delta_{3}$ are given, respectively, by

$$
a_{1}=Q x, \quad a_{2}=0, \quad q_{c}=1+f_{1}, \quad \hat{c}_{c}^{2}=0, \quad \Delta_{3}=\Delta_{1}+Q x,
$$

where

$$
x \equiv \frac{\dot{\phi}}{M_{\mathrm{pl}} H} .
$$

Since the coupling $f_{2}$ is absent, $\hat{c}_{c}^{2}$ vanishes. From Eqs. (5.24)-(5.27), it follows that

$$
\begin{array}{ll}
G_{c c}=\frac{G}{Q_{t}}\left(1+f_{1}\right)\left[1+\frac{\left(Q x+\Delta_{1}\right)^{2}}{\Delta_{2}}\right], & G_{c b}=\frac{G}{Q_{t}}\left[1+\frac{\Delta_{1}\left(Q x+\Delta_{1}\right)}{\Delta_{2}}\right], \\
G_{b c}=\frac{G}{Q_{t}}\left(1+f_{1}\right)\left[1+\frac{\Delta_{1}\left(Q x+\Delta_{1}\right)}{\Delta_{2}}\right], & G_{b b}=\frac{G}{Q_{t}}\left(1+\frac{\Delta_{1}^{2}}{\Delta_{2}}\right) .
\end{array}
$$

As long as the stability conditions of tensor and scalar perturbations are satisfied, it follows that $Q_{t}>0, q_{c}=1+f_{1}>0$, and $\Delta_{2}>0$. Hence both $G_{c c}$ and $G_{b b}$ are positive, with the positive scalar-matter interactions characterized by $\left(Q x+\Delta_{1}\right)^{2} / \Delta_{2}>0$ and $\Delta_{1}^{2} / \Delta_{2}>0$. According to our knowledge, the gravitational couplings (5.32) have not been explicitly derived in coupled Horndeski theories given by the action (2.2).

In the following, we specify the scalar-graviton sector to the minimally coupled k-essence given by the action,

$$
\mathcal{S}_{\mathrm{H}}=\int \mathrm{d}^{4} x \sqrt{-g}\left[\frac{M_{\mathrm{pl}}^{2}}{2} R+G_{2}(\phi, X)\right] .
$$


We also consider a light-mass scalar field satisfying

$$
\frac{q_{t} a^{2} M_{\phi}^{2}}{q_{s} \hat{c}_{s}^{2} k^{2}} \ll 1
$$

in $\Delta_{2}$ of Eq. (5.14). We also adopt the condition (5.34) for the theories discussed later in Secs. V B 2 and V B 3. Then, we have

$$
Q_{t}=1, \quad q_{s} \hat{c}_{s}^{2}=2 M_{\mathrm{pl}}^{2} G_{2, X}, \quad \alpha_{\mathrm{M}}=0, \quad \alpha_{\mathrm{B}}=0, \quad \Delta_{1}=0, \quad \Delta_{2}=\frac{x^{2}}{2} G_{2, X} .
$$

From Eq. (5.32), we obtain

$$
G_{c c}=G\left(1+f_{1}\right)\left(1+\frac{2 Q^{2}}{G_{2, X}}\right), \quad G_{c b}=G, \quad G_{b c}=G\left(1+f_{1}\right), \quad G_{b b}=G .
$$

The coupling term $2 Q^{2} / G_{2, X}$ in $G_{c c}$ coincides with that derived in Refs. [86, 92]. For a canonical scalar field given by the Lagrangian $G_{2}=X-V(\phi)$, the scalar-matter interaction in $G_{c c}$ reduces to the well-known form $2 Q^{2}$ [28, 93], which enhances the growth rate of CDM perturbations.

\section{Theories with $f_{1}=f_{1}(\phi)$ and $f_{2}=f_{2}\left(n_{c}, \phi\right)$}

Besides the nonminimal coupling $f_{1}=f_{1}(\phi)$, we consider the case in which the second interaction of the form $f_{2}=f_{2}\left(n_{c}, \phi\right)$ is present. For concreteness, we focus on the couplings,

$$
f_{1}=f_{1}(\phi), \quad f_{2}=c n_{c}^{-1}+F_{2}(\phi)
$$

where $f_{1}, F_{2}$ are functions of $\phi$, and $c$ is a constant. In this case, we have $\hat{c}_{c}^{2}=0$. Together with the coupling $Q$ defined in Eq. (5.29), we introduce the following dimensionless quantities,

$$
q=\frac{M_{\mathrm{pl}} Q_{, \phi}}{Q}, \quad \Omega_{f_{2}}=\frac{c \dot{\phi}}{3 M_{\mathrm{pl}}^{2} H^{2}}
$$

and consider a light scalar field whose mass $M_{\phi}$ is in the range (5.34).

For the scalar-graviton sector, we adopt a canonical scalar field given by the action

$$
\mathcal{S}_{\mathrm{H}}=\int \mathrm{d}^{4} x \sqrt{-g}\left[\frac{M_{\mathrm{pl}}^{2}}{2} R+X-V(\phi)\right]
$$

where $V(\phi)$ is the field potential. Then, it follows that

$$
a_{1}=\frac{\left(1+f_{1}\right) \Omega_{c} Q x+\left(3+\epsilon_{\phi}\right) \Omega_{f_{2}}}{\left(1+f_{1}\right) \Omega_{c}+\Omega_{f_{2}}}, \quad a_{2}=-\frac{\Omega_{f_{2}}}{\left(1+f_{1}\right) \Omega_{c}+\Omega_{f_{2}}}, \quad q_{c}=1+f_{1}+\frac{\Omega_{f_{2}}}{\Omega_{c}},
$$

and

$$
\begin{aligned}
& \alpha_{\mathrm{M}}=0, \quad \alpha_{\mathrm{B}}=0, \quad \Delta_{1}=0, \quad \Delta_{2}=\frac{x^{2}}{2}, \quad \Delta_{3}=\frac{\left(1+f_{1}\right) \Omega_{c} Q x}{\left(1+f_{1}\right) \Omega_{c}+\Omega_{f_{2}}} \\
& \epsilon_{\Delta_{1}}=0, \quad \epsilon_{\Delta_{2}}=2\left(\epsilon_{\phi}-\epsilon_{H}\right), \quad \epsilon_{\Delta_{3}}=\frac{\left(1+f_{1}\right) \Omega_{c}\left(\epsilon_{\phi}-\epsilon_{H}+q x\right)+\Omega_{f_{2}}\left[(Q+q) x-3-\epsilon_{H}\right]}{\left(1+f_{1}\right) \Omega_{c}+\Omega_{f_{2}}} .
\end{aligned}
$$

The quantity $x$, which is defined by Eq. (5.31), is expressed as $x=\epsilon \sqrt{2 \Delta_{2}}$, where $\epsilon=+1$ for $\dot{\phi}>0$ and $\epsilon=-1$ for $\dot{\phi}<0$. The effective gravitational couplings (5.24)-(5.27) reduce, respectively, to

$$
\begin{aligned}
G_{c c} & =G\left(1+f_{1}\right)\left[1+2 Q^{2}-\Omega_{f_{2}} \frac{2 \Delta_{2}(1-2 Q q)-4 \epsilon Q \sqrt{2 \Delta_{2}}+3 \Omega_{f_{2}}\left(1+2 Q^{2}\right)}{2 \Omega_{c} \Delta_{2}\left(1+f_{1}\right)+\Omega_{f_{2}}\left(2 \Delta_{2}+3 \Omega_{f_{2}}\right)}\right], \\
G_{c b} & =G \frac{2 \Omega_{c}\left(1+f_{1}\right) \Delta_{2}}{2\left[\Omega_{c}\left(1+f_{1}\right)+\Omega_{f_{2}}\right] \Delta_{2}+3 \Omega_{f_{2}}^{2}}, \quad G_{b c}=G\left(1+f_{1}\right), \quad G_{b b}=G .
\end{aligned}
$$


Compared to the values given in Eq. (5.36), the $n_{c}$ dependence in $f_{2}$ modifies both $G_{c c}$ and $G_{c b}$. We note that there is no modification to the gravitational couplings from the term $F_{2}(\phi)$ in $f_{2}$.

If we consider the theories with [50]

$$
f_{1}=0
$$

i.e., $Q=0$, Eq. (5.42) gives

$$
G_{c c}=G_{c b}=G \frac{2 \Omega_{c} \Delta_{2}}{2\left(\Omega_{c}+\Omega_{f_{2}}\right) \Delta_{2}+3 \Omega_{f_{2}}^{2}}, \quad G_{b c}=G_{b b}=G,
$$

where $G_{c c}$ matches with that derived in Eq. [59]. Since $\Delta_{2}>0$ and $\Omega_{c}>0$, the gravitational couplings with CDM can be smaller than $G$ (i.e., $G_{c c}=G_{c b}<G$ ) for

$$
\Omega_{f_{2}}>0, \text { i.e., } \quad c \dot{\phi}>0 \text {. }
$$

This is an explicit example in which the $n_{c}$ dependence in $f_{2}$ allows the possibility for realizing weak gravity on scales relevant to the linear growth of large-scale structures. We note that, under the absence of ghosts and Laplacian instabilities, the effective gravitational coupling in uncoupled Horndeski theories is destined to be larger than $G$ $[19,64]$. Hence, the coupled model with weak gravitational interactions can be observationally distinguished from uncoupled Horndeski theories. It is of interest to explore further whether the coupled dark energy model with the coupling $f_{2} \propto n_{c}^{-1}$ can be in better fit to the observational data in comparison to the $\Lambda$ CDM model. In the presence of nonvanishing $f_{1}(\phi)$, the gravitational couplings $G_{c c}$ and $G_{c b}$ in Eq. (5.42) differ from those in Eq. (5.44), so the observational signatures are different.

$$
\text { 3. Theories with } f_{1}=0 \text { and } f_{2}=f_{2}\left(n_{c}, X\right)
$$

Finally, we study the effect of $X$-dependence in $f_{2}$ on the effective gravitational couplings. For concreteness, let us consider the functions,

$$
f_{1}=0, \quad f_{2}=\beta n_{c}^{-1} X^{s},
$$

where $\beta$ and $s$ are constants. For this choice of $f_{2}, \hat{c}_{c}^{2}$ vanishes. On using the definition of fluid four-velocity (2.6), the interacting action (2.4) can be written as

$$
\mathcal{S}_{\text {int }}=\int \mathrm{d}^{4} x \sqrt{-g} \beta X^{s} u_{c}^{\mu} \partial_{\mu} \phi .
$$

On the flat FLRW background (2.10) with $N=1$, the term $u_{c}^{\mu} \partial_{\mu} \phi$ is equivalent to $\dot{\phi}$. Then, the above interacting Lagrangian is proportional to $\dot{\phi}^{2 s+1}$. For the scalar-graviton sector, we adopt the minimally coupled k-essence described by the action (5.33). Then, Eqs. (2.38) and (2.39) reduce, respectively, to

$$
\begin{aligned}
& \rho_{\mathrm{DE}}=-G_{2}+\dot{\phi}^{2} G_{2, X}+2 s \bar{\beta} \dot{\phi}^{2 s+1}, \\
& P_{\mathrm{DE}}=G_{2}+\bar{\beta} \dot{\phi}^{2 s+1},
\end{aligned}
$$

where $\bar{\beta}=2^{-s} \beta$. For the power,

$$
s=\frac{1}{2}
$$

the last terms in Eqs. (5.48) and (5.49) are proportional to $\dot{\phi}^{2}$, i.e., the same time dependence as the standard kinetic term $X$ in $G_{2}$. If $s \neq 1 / 2$, the last terms in Eqs. (5.48) and (5.49) can dominate over $X$ either in the asymptotic past or future. In the following, we focus on the model with the power (5.50). Then, it follows that

$$
a_{1}=\frac{\bar{\beta} x^{2}\left(3+2 \epsilon_{\phi}\right)}{3 \Omega_{c}+\bar{\beta} x^{2}}, \quad a_{2}=-\frac{\bar{\beta} x^{2}}{3 \Omega_{c}+\bar{\beta} x^{2}}, \quad q_{c}=1+\frac{\bar{\beta} x^{2}}{3 \Omega_{c}}, \quad \hat{c}_{c}^{2}=0,
$$

where $x$ is defined by Eq. (5.31), and

$$
\begin{array}{ll}
Q_{t}=1, & q_{s} \hat{c}_{s}^{2}=2 M_{\mathrm{pl}}^{2}\left(G_{2, X}+\bar{\beta}\right) \\
\Delta_{1}=0, & \alpha_{\mathrm{M}}=0, \quad \alpha_{\mathrm{B}}=0, \\
\Delta_{2} & =\frac{x^{2}}{2}\left(G_{2, X}+\bar{\beta}\right), \quad \Delta_{3}=0 .
\end{array}
$$


Substituting Eqs. (5.51) and (5.52) into (5.24)-(5.27), we obtain

$$
\begin{aligned}
G_{c c} & =G_{c b}=G\left(1+\frac{G_{2, X}+2 \bar{\beta}}{G_{2, X}+\bar{\beta}} \frac{\bar{\beta} x^{2}}{3 \Omega_{c}}\right)^{-1}, \\
G_{b c} & =G_{b b}=1 .
\end{aligned}
$$

From Eq. (5.51), the ghost does not appear from the CDM sector for $\bar{\beta}>0$. Moreover, the condition $G_{2, X}+\bar{\beta}>0$ is required for the positivity of $q_{s} \hat{c}_{s}^{2}$ in Eq. (5.52). Then, the second term in the parenthesis of Eq. (5.53) is positive, so that $G_{c c}=G_{c b}<G$. This is the explicit example in which the $X$-dependence in $f_{2}$ leads to the realization of weak gravity on cosmological scales.

The interacting action (5.47) with the power (5.50) can be further generalized to the theories containing the nonlinear dependence of $u_{c}^{\mu} \partial_{\mu} \phi$, e.g.,

$$
\mathcal{S}_{\text {int }}=\int \mathrm{d}^{4} x \sqrt{-g} \beta X^{s}\left(u_{c}^{\mu} \partial_{\mu} \phi\right)^{2-2 s} .
$$

By setting $s=0$, the interacting action (5.55) recovers the theory studied in Ref. [52] as a special case. The detailed analysis of cosmological dynamics for the extended coupling (5.55) is given in Ref. [94] (which was submitted one month after the initial submission of this paper).

\section{CONCLUSIONS}

We studied the interacting dark energy scenario in which a scalar field $\phi$ is coupled to the CDM perfect fluid given by the Schutz-Sorkin action (2.3). The scalar-graviton sector is described by the Horndeski action (2.2) with the tensor propagation speed squared equivalent to that of light. We considered the new interacting action (2.4) containing the $X$ dependence in the couplings $f_{1}$ and $f_{2}$. Our analysis is sufficiently general in that it accommodates a wide variety of nonminimal and derivative couplings studied in the literature [23, 25, 49, 50, 59, 72]. Moreover, unlike most of past related papers, we did not restrict the dark energy field to quintessence or k-essence.

In Sec. II, we derived the background equations of motion on the flat FLRW background in the forms (2.20)-(2.22). As long as the quantity $q_{s}$ defined by Eq. (2.31) does not vanish, the dynamical system can be solved for $\dot{H}$ and $\ddot{\phi}$. Indeed, the stability analysis performed in Sec. IV leads to the condition $q_{s}>0$ to avoid the ghost associated with the scalar perturbation. We also identified the total CDM density $\hat{\rho}_{c}$ and pressure $\hat{P}_{c}$ containing the effect of interactions with the scalar field, as Eqs. (2.32) and (2.33). We showed that CDM interacts with dark energy according to Eqs. (2.35) and (2.40), whose signs on the right hand sides are opposite to each other.

In Sec. III, we expanded the action up to second order in scalar perturbations without fixing any gauge conditions. We explicitly computed the quadratic-order actions arising from $\mathcal{S}_{\mathrm{H}}, \mathcal{S}_{M}$, $\mathcal{S}_{\text {int }}$ and finally took the sum of them on account of the background equations. The final second-order action $\mathcal{S}_{s}^{(2)}$ is of the form $(3.37)$, where $L_{\text {int }}$ is the Lagrangian arising from the couplings $f_{1}$ and $f_{2}$. In Sec. III B, we also obtained the full linear perturbation equations of motion in the gauge-ready form, i.e., ready for choosing any particular gauges.

In Sec. IV, we first introduced a number of gauge-invariant perturbed quantities and discussed several different gauge choices. In the flat and unitary gauges, we derived the second-order actions of dynamical scalar perturbations after eliminating nondynamical quantities and identified stability conditions in the small-scale limit. The conditions for the absence of ghosts and Laplacian instabilities are independent of the gauge choices. The ghosts are absent under the conditions (4.18) and (4.19). For CDM satisfying $\hat{c}_{c}^{2}=0$, the off-diagonal components of matrix $\boldsymbol{B}$ do not modify the CDM sound speed, i.e., $c_{\mathrm{CDM}}^{2}=0$, but they affect the propagation speed squared $c_{s}^{2}$ of the scalar degree of freedom. We showed that $c_{s}^{2}$ is the sum of $\hat{c}_{s}^{2}$ and $\Delta c_{s}^{2}$, whose explicit expressions are given, respectively, by Eqs. (4.40) and (4.41).

In Sec. V, we derived the effective gravitational couplings for CDM and baryon density perturbations by using the quasi-static approximation for the modes deep inside the sound horizon. We obtained the Bardeen gravitational potentials $\Psi, \Phi$, and the field perturbation $\delta \phi_{\mathrm{N}}$ in the gauge-independent manner. The difference from the uncoupled case is that the time derivative $\dot{\delta}_{c \mathrm{~N}}$ appears in the expressions of $\Psi, \Phi, \delta \phi_{\mathrm{N}}$ given by Eqs. (5.15)-(5.17). Taking the time derivative of $\delta \phi_{\mathrm{N}}$ in Eq. (5.17) gives rise to the second derivative $\ddot{\delta}_{c \mathrm{~N}}$, so we need to solve Eq. (5.7) for $\ddot{\delta}_{c \mathrm{~N}}$ to obtain the closed-form equation for $\delta_{c \mathrm{~N}}$. As a result, the CDM and baryon density contrasts obey Eqs. (5.22) and (5.23), respectively, with the effective gravitational couplings (5.24)-(5.27).

We applied our general formulas of $G_{c c}, G_{c b}, G_{b c}, G_{b b}$ to three different forms of couplings and discussed how they reproduce the effective gravitational couplings known in the literature. In particular, the $n_{c}$ or $X$ dependence in $f_{2}$ offers an interesting possibility for realizing $G_{c c}$ and $G_{c b}$ smaller than $G$. In uncoupled Horndeski theories, the 
gravitational couplings are usually larger than $G$. Moreover, the presence of matter couplings $f_{1}$ and $f_{2}$ gives rise to the values of $G_{c c}, G_{c b}, G_{b c}, G_{b b}$ generally different from each other, while this is not the case in uncoupled Horndeski theories. These properties show that the coupled theories with weak gravitational interactions can be observationally distinguished from uncoupled theories.

In this paper we did not construct particular models of coupled dark energy, but it is of interest to do so to observationally probe the signature of interactions with CDM. First of all, theoretically consistent models need to satisfy all the small-scale stability conditions derived in Sec. IV. The next step is to predict observational signatures of models both at the background and perturbation levels, e.g., the dark energy and CDM equations of state and the growth of perturbations. Then, the models should be confronted with numerous observational data associated with the cosmic expansion and growth histories. These interesting issues are left for future works.

\section{Acknowledgements}

RK is supported by the Grant-in-Aid for Young Scientists B of the JSPS No.17K14297. ST is supported by the Grant-in-Aid for Scientific Research Fund of the JSPS No.19K03854 and MEXT KAKENHI Grant-in-Aid for Scientific Research on Innovative Areas "Cosmic Acceleration" (No. 15H05890).

[1] E. J. Copeland, M. Sami and S. Tsujikawa, Int. J. Mod. Phys. D 15, 1753 (2006) [hep-th/0603057].

[2] T. Clifton, P. G. Ferreira, A. Padilla and C. Skordis, Phys. Rept. 513, 1 (2012) [arXiv:1106.2476 [astro-ph.CO]].

[3] A. Joyce, B. Jain, J. Khoury and M. Trodden, Phys. Rept. 568, 1 (2015) [arXiv:1407.0059 [astro-ph.CO]].

[4] G. Jungman, M. Kamionkowski and K. Griest, Phys. Rept. 267, 195 (1996) [hep-ph/9506380].

[5] G. Bertone, D. Hooper and J. Silk, Phys. Rept. 405, 279 (2005) [hep-ph/0404175].

[6] P. A. R. Ade et al. [Planck Collaboration], Astron. Astrophys. 594, A13 (2016) [arXiv:1502.01589 [astro-ph.CO]].

[7] A. G. Riess et al., Astrophys. J. 826, 56 (2016) [arXiv:1604.01424 [astro-ph.CO]].

[8] H. Hildebrandt et al., Mon. Not. Roy. Astron. Soc. 465, 1454 (2017) [arXiv:1606.05338 [astro-ph.CO]].

[9] G. W. Horndeski, Int. J. Theor. Phys. 10, 363 (1974).

[10] C. Deffayet, X. Gao, D. A. Steer and G. Zahariade, Phys. Rev. D 84, 064039 (2011) [arXiv:1103.3260 [hep-th]].

[11] T. Kobayashi, M. Yamaguchi and J. Yokoyama, Prog. Theor. Phys. 126, 511 (2011) [arXiv:1105.5723 [hep-th]].

[12] C. Charmousis, E. J. Copeland, A. Padilla and P. M. Saffin, Phys. Rev. Lett. 108, 051101 (2012) [arXiv:1106.2000 [hep-th]].

[13] B. P. Abbott et al. [LIGO Scientific and Virgo Collaborations], Phys. Rev. Lett. 119, 161101 (2017) [arXiv:1710.05832 $[\mathrm{gr}-\mathrm{qc}]]$.

[14] L. Lombriser and A. Taylor, JCAP 1603, 031 (2016) [arXiv:1509.08458 [astro-ph.CO]].

[15] P. Creminelli and F. Vernizzi, Phys. Rev. Lett. 119, 251302 (2017) [arXiv:1710.05877 [astro-ph.CO]].

[16] J. M. Ezquiaga and M. Zumalacarregui, Phys. Rev. Lett. 119, 251304 (2017) [arXiv:1710.05901 [astro-ph.CO]].

[17] J. Sakstein and B. Jain, Phys. Rev. Lett. 119, 251303 (2017) [arXiv:1710.05893 [astro-ph.CO]].

[18] T. Baker, E. Bellini, P. G. Ferreira, M. Lagos, J. Noller and I. Sawicki, Phys. Rev. Lett. 119, 251301 (2017) [arXiv:1710.06394 [astro-ph.CO]].

[19] L. Amendola, M. Kunz, I. D. Saltas and I. Sawicki, Phys. Rev. Lett. 120, 131101 (2018) [arXiv:1711.04825 [astro-ph.CO]].

[20] R. Kase and S. Tsujikawa, Phys. Rev. D 97, 103501 (2018) [arXiv:1802.02728 [gr-qc]].

[21] S. Peirone, G. Benevento, N. Frusciante and S. Tsujikawa, Phys. Rev. D 100, 063540 (2019) [arXiv:1905.05166 [astroph.CO]].

[22] S. Peirone, G. Benevento, N. Frusciante and S. Tsujikawa, Phys. Rev. D 100, 063509 (2019) [arXiv:1905.11364 [astroph.CO]].

[23] C. Wetterich, Astron. Astrophys. 301, 321 (1995) [hep-th/9408025].

[24] L. Amendola, Phys. Rev. D 60, 043501 (1999) [astro-ph/9904120].

[25] L. Amendola, Phys. Rev. D 62, 043511 (2000) [astro-ph/9908023].

[26] B. Gumjudpai, T. Naskar, M. Sami and S. Tsujikawa, JCAP 0506, 007 (2005) [hep-th/0502191].

[27] L. Amendola, M. Quartin, S. Tsujikawa and I. Waga, Phys. Rev. D 74, 023525 (2006) [astro-ph/0605488].

[28] P. A. R. Ade et al. [Planck Collaboration], Astron. Astrophys. 594, A14 (2016) [arXiv:1502.01590 [astro-ph.CO]].

[29] N. Dalal, K. Abazajian, E. E. Jenkins and A. V. Manohar, Phys. Rev. Lett. 87, 141302 (2001) [astro-ph/0105317].

[30] W. Zimdahl and D. Pavon, Phys. Lett. B 521, 133 (2001) [astro-ph/0105479].

[31] L. P. Chimento, A. S. Jakubi, D. Pavon and W. Zimdahl, Phys. Rev. D 67, 083513 (2003) [astro-ph/0303145].

[32] B. Wang, Y. g. Gong and E. Abdalla, Phys. Lett. B 624, 141 (2005) [hep-th/0506069].

[33] H. Wei and S. N. Zhang, Phys. Lett. B 644, 7 (2007) [astro-ph/0609597].

[34] L. Amendola, G. Camargo Campos and R. Rosenfeld, Phys. Rev. D 75, 083506 (2007) [astro-ph/0610806].

[35] Z. K. Guo, N. Ohta and S. Tsujikawa, Phys. Rev. D 76, 023508 (2007) [astro-ph/0702015 [astro-ph]].

[36] J. Valiviita, E. Majerotto and R. Maartens, JCAP 0807, 020 (2008) [arXiv:0804.0232 [astro-ph]].

[37] J. Valiviita, R. Maartens and E. Majerotto, Mon. Not. Roy. Astron. Soc. 402, 2355 (2010) [arXiv:0907.4987 [astro-ph.CO]]. 
[38] M. B. Gavela, D. Hernandez, L. Lopez Honorez, O. Mena and S. Rigolin, JCAP 0907, 034 (2009) [arXiv:0901.1611 [astro-ph.CO]].

[39] B. M. Jackson, A. Taylor and A. Berera, Phys. Rev. D 79, 043526 (2009) [arXiv:0901.3272 [astro-ph.CO]].

[40] V. Faraoni, J. B. Dent and E. N. Saridakis, Phys. Rev. D 90, 063510 (2014) [arXiv:1405.7288 [gr-qc]].

[41] V. Salvatelli, N. Said, M. Bruni, A. Melchiorri and D. Wands, Phys. Rev. Lett. 113, 181301 (2014) [arXiv:1406.7297 [astro-ph.CO]].

[42] B. Wang, E. Abdalla, F. Atrio-Barandela and D. Pavon, Rept. Prog. Phys. 79, 096901 (2016) [arXiv:1603.08299 [astroph.CO]].

[43] E. Di Valentino, A. Melchiorri and O. Mena, Phys. Rev. D 96, 043503 (2017) [arXiv:1704.08342 [astro-ph.CO]].

[44] E. Di Valentino, A. Melchiorri, O. Mena and S. Vagnozzi, arXiv:1908.04281 [astro-ph.CO].

[45] Z. Berezhiani, A. D. Dolgov and I. I. Tkachev, Phys. Rev. D 92, 061303 (2015) [arXiv:1505.03644 [astro-ph.CO]].

[46] A. Chudaykin, D. Gorbunov and I. Tkachev, Phys. Rev. D 97, 083508 (2018) [arXiv:1711.06738 [astro-ph.CO]].

[47] N. Tamanini, Phys. Rev. D 92, 043524 (2015) [arXiv:1504.07397 [gr-qc]].

[48] A. Pourtsidou, C. Skordis and E. J. Copeland, Phys. Rev. D 88, 083505 (2013) [arXiv:1307.0458 [astro-ph.CO]].

[49] C. G. Boehmer, N. Tamanini and M. Wright, Phys. Rev. D 91, 123002 (2015) [arXiv:1501.06540 [gr-qc]].

[50] C. G. Boehmer, N. Tamanini and M. Wright, Phys. Rev. D 91, 123003 (2015) [arXiv:1502.04030 [gr-qc]].

[51] C. Skordis, A. Pourtsidou and E. J. Copeland, Phys. Rev. D 91, 083537 (2015) [arXiv:1502.07297 [astro-ph.CO]].

[52] A. Pourtsidou and T. Tram, Phys. Rev. D 94, 043518 (2016) [arXiv:1604.04222 [astro-ph.CO]].

[53] M. S. Linton, A. Pourtsidou, R. Crittenden and R. Maartens, JCAP 1804 (2018) 043 [arXiv:1711.05196 [astro-ph.CO]].

[54] B. F. Schutz and R. Sorkin, Annals Phys. 107, 1 (1977).

[55] J. D. Brown, Class. Quant. Grav. 10, 1579 (1993) [gr-qc/9304026].

[56] A. De Felice, J. M. Gerard and T. Suyama, Phys. Rev. D 81, 063527 (2010) [arXiv:0908.3439 [gr-qc]].

[57] D. Bettoni, S. Liberati and L. Sindoni, JCAP 1111, 007 (2011) [arXiv:1108.1728 [gr-qc]].

[58] D. Bettoni and S. Liberati, JCAP 1508, 023 (2015) [arXiv:1502.06613 [gr-qc]].

[59] T. S. Koivisto, E. N. Saridakis and N. Tamanini, JCAP 1509, 047 (2015) [arXiv:1505.07556 [astro-ph.CO]].

[60] A. De Felice, L. Heisenberg, R. Kase, S. Mukohyama, S. Tsujikawa and Y. l. Zhang, JCAP 1606, 048 (2016) [arXiv:1603.05806 [gr-qc]].

[61] J. Dutta, W. Khyllep and N. Tamanini, Phys. Rev. D 93, 063004 (2016) [arXiv:1602.06113 [gr-qc]].

[62] J. Dutta, W. Khyllep and N. Tamanini, JCAP 1801, 038 (2018) [arXiv:1707.09246 [gr-qc]].

[63] R. Kase and S. Tsujikawa, JCAP 1811, 024 (2018) [arXiv:1805.11919 [gr-qc]].

[64] R. Kase and S. Tsujikawa, Int. J. Mod. Phys. D 28, 1942005 (2019) [arXiv:1809.08735 [gr-qc]].

[65] N. Frusciante, R. Kase, N. J. Nunes and S. Tsujikawa, Phys. Rev. D 98, 123517 (2018) [arXiv:1810.07957 [gr-qc]].

[66] N. Frusciante, R. Kase, K. Koyama, S. Tsujikawa and D. Vernieri, Phys. Lett. B 790, 167 (2019) [arXiv:1812.05204 [gr-qc]].

[67] S. Nakamura, R. Kase and S. Tsujikawa, arXiv:1907.12216 [gr-qc].

[68] F. Beutler et al. [BOSS Collaboration], Mon. Not. Roy. Astron. Soc. 443, 1065 (2014) [arXiv:1312.4611 [astro-ph.CO]].

[69] L. Samushia et al., Mon. Not. Roy. Astron. Soc. 439, 3504 (2014) [arXiv:1312.4899 [astro-ph.CO]].

[70] E. Macaulay, I. K. Wehus and H. K. Eriksen, Phys. Rev. Lett. 111, 161301 (2013) [arXiv:1303.6583 [astro-ph.CO]].

[71] A. Vikhlinin et al., Astrophys. J. 692, 1060 (2009) [arXiv:0812.2720 [astro-ph]].

[72] B. J. Barros, Phys. Rev. D 99, 064051 (2019) [arXiv:1901.03972 [gr-qc]].

[73] J. Gleyzes, D. Langlois, M. Mancarella and F. Vernizzi, JCAP 1508, 054 (2015) [arXiv:1504.05481 [astro-ph.CO]].

[74] R. Kimura, T. Suyama, M. Yamaguchi, D. Yamauchi and S. Yokoyama, Publ. Astron. Soc. Jap. 70, 5 (2018) [arXiv:1709.09371 [astro-ph.CO]].

[75] F. Chibana, R. Kimura, M. Yamaguchi, D. Yamauchi and S. Yokoyama, arXiv:1908.07173 [astro-ph.CO].

[76] J. c. Hwang and H. r. Noh, Phys. Rev. D 65, 023512 (2002) [astro-ph/0102005].

[77] L. Heisenberg, R. Kase and S. Tsujikawa, Phys. Rev. D 98, 123504 (2018) [arXiv:1807.07202 [gr-qc]].

[78] J. M. Bardeen, Phys. Rev. D 22, 1882 (1980).

[79] H. Kodama and M. Sasaki, Prog. Theor. Phys. Suppl. 78, 1 (1984).

[80] V. F. Mukhanov, H. A. Feldman and R. H. Brandenberger, Phys. Rept. 215, 203 (1992).

[81] V. N. Lukash, Sov. Phys. JETP 52, 807 (1980).

[82] D. H. Lyth, Phys. Rev. D 31, 1792 (1985).

[83] V. F. Mukhanov, JETP Lett. 41, 493 (1985).

[84] M. Sasaki, Prog. Theor. Phys. 76, 1036 (1986).

[85] B. Boisseau, G. Esposito-Farese, D. Polarski and A. A. Starobinsky, Phys. Rev. Lett. 85, 2236 (2000) [gr-qc/0001066].

[86] S. Tsujikawa, Phys. Rev. D 76, 023514 (2007) [arXiv:0705.1032 [astro-ph]].

[87] A. De Felice, T. Kobayashi and S. Tsujikawa, Phys. Lett. B 706, 123 (2011) [arXiv:1108.4242 [gr-qc]].

[88] W. Hu and I. Sawicki, Phys. Rev. D 76, 064004 (2007) [arXiv:0705.1158 [astro-ph]].

[89] A. A. Starobinsky, JETP Lett. 86, 157 (2007) [arXiv:0706.2041 [astro-ph]].

[90] S. Tsujikawa, Phys. Rev. D 77, 023507 (2008) [arXiv:0709.1391 [astro-ph]].

[91] H. Sandvik, M. Tegmark, M. Zaldarriaga and I. Waga, Phys. Rev. D 69, 123524 (2004) [astro-ph/0212114].

[92] L. Amendola, Phys. Rev. Lett. 93, 181102 (2004) [hep-th/0409224].

[93] L. Amendola, Phys. Rev. D 69, 103524 (2004) [astro-ph/0311175].

[94] R. Kase and S. Tsujikawa, arXiv:1911.02179 [gr-qc]. 